\title{
Models for Assembly Line Balancing by temporal, spatial and
}

\section{ergonomic risk attributes}

\author{
Joaquín Bautista $^{1, *}$, Cristina Batalla-García ${ }^{1}$, Rocío Alfaro-Pozo ${ }^{1}$
}

\begin{abstract}
Assembly lines with mixed products present ergonomic risks that can affect productivity of workers and lines. Because of that, the line balancing must consider the risk of injury in regard with the set of tasks necessary to process a product unit, in addition to other managerial and technological attributes such as the workload or the space. Therefore, in this paper we propose a new approach to solve the assembly line balancing problem considering temporal, spatial and ergonomic attributes at once. We formulate several mathematical models and we analyze the behavior of one of these models through case study linked to Nissan. Furthermore, we study the effect of the demand plan variations and ergonomic risk on the line balancing result.
\end{abstract}

Keywords: Manufacturing; Ergonomic risks; Flexible manufacturing systems; Assembly line balancing; Linear programming.

\section{Introduction}

Manufacturing and/or assembly lines are common in product-oriented production systems. This is the case of the automotive sector, where the use of the same line to process different product types is very common. In such cases, the products although be similar, differ in the use of resources and components' consumption. For that reason, once the product, the process, and the line layout configuration have been established, the first step to design a mixed-product assembly line is to average the processing times of operations that are required by the different product types, according to the proportions of each product type in the demand plan. Then, the second design decision is the line balancing.

\footnotetext{
${ }^{1}$ ETSEIB UPC. Universitat Politècnica de Catalunya. Avda. Diagonal 647, 08028 Barcelona, Spain.

${ }^{*}$ Corresponding author. Tel.: +34 93 4011703; fax: +34 934016054. E-mail addresses: joaquin.bautista@upc.edu (J.Bautista), cristina.batalla@upc.edu (C.Batalla),rocio.alfaro@upc.edu (R.Alfaro).
} 
The Assembly Line Balancing Problem $(A L B P)$ is a classic problem from literature (Salveson, 1955).The problem focuses on assigning the set of elementary tasks, necessary to assemble or disassemble a product (e.g., engines, batteries, cars), to the set of workstations or modules that compose the line, consistently and efficiently. These workstations (commonly associated with teams of workers and/or robots) are typically arranged in series, one behind another, and connected by a transport system that allows the movement of the work in progress at a constant speed. Thus, each workstation has a constant time (cycle time, $c$ ) to complete the assigned workload.

Depending on the constraints taken into account, the problem can be divided. Indeed, Baybars (1986) classified the $A L B P$ family into two types of problems:

- The Simple Assembly Line Balancing Problem (SALBP).

- The General Assembly Line Balancing Problem (GALBP).

The $S A L B P$ class contains assembly problems that attempt to minimize the total idle time when two types of task assignment constraints are exclusively considered:

1) Cumulative constraints associated with the available work time at workstations.

2) Precedence constraints established by the order in which the tasks must be executed. On the other hand, the GALBP class (Becker and Scholl, 2006) contains problems with additional considerations, such as (1) the restricted assignment of tasks (Scholl et al., 2010); or (2) the assignment in block of certain tasks (Battaiia and Dolgui, 2012).

However the original problems have been extended in the literature in the last decades (Battaïa and Dolgui, 2013), resulting in problems that consider, in addition to the cycle time $(c)$ and the number of workstations $(m)$, other attributes, such as spatial conditions and ergonomic parameters.

Problems that consider the space or area $(A)$ available for materials and tools at each workstation are included in the family problems whose name is Time and Space Constrained Assembly Line Balancing Problems (TSALBP) (Chica et. al., 2010; Chica et. al., 2011). Given a set $J$ of $|J|$ tasks, with their temporal $t_{j}$ and spatial $a_{j}$ attributes $(\forall j=1, \ldots,|J|)$ and a precedence graph, these problems focus on assigning each task to a single workstation, such that:

1) All precedence constraints are satisfied.

2) No workstation with workload time greater than the cycle time, $(c)$.

3) None workstation requires an area greater than the available area per station $(A)$. 
In short, considering the incorporation of the different attributes of tasks defined above into the balancing problems and the optimization criterion, both families of problems, SALBP and TSALBP (Bautista and Pereira, 2007), include a set of four and eight problem types, respectively (Table 1).

Table 1. SALBP and TSALBP typology.

\begin{tabular}{l|cccc}
\hline \multicolumn{1}{c|}{ Name } & $m$ & $c$ & $A$ & Type \\
\hline SALBP-F & Given & Given & - & $\mathrm{F}$ \\
SALBP-1 & Minimize & Given & - & OP \\
SALBP-2 & Given & Minimize & - & OP \\
$S A L B P-E$ & Minimize & Minimize & - & OP \\
$T S A L B P-F$ & Given & Given & Given & $\mathrm{F}$ \\
$T S A L B P-m$ & Minimize & Given & Given & OP \\
$T S A L B P-c$ & Given & Minimize & Given & OP \\
$T S A L B P-A$ & Given & Given & Minimize & OP \\
$T S A L B P-m / c$ & Minimize & Minimize & Given & MOP \\
$T S A L B P-m / A$ & Minimize & Given & Minimize & MOP \\
$T S A L B P-c / A$ & Given & Minimize & Minimize & MOP \\
$T S A L B P-m / c / A$ & Minimize & Minimize & Minimize & MOP \\
\hline
\end{tabular}

For both typologies, the column "Type" indicates if the problem is one of feasibility (F), mono-objective (OP) or multi-objective (MOP); and the columns " $m$ ", " $c$ " and " $A$ " indicate if these attributes are variables (Minimize) or parameters (Given). It should be noted that $S A L B P$ family do not consider the spatial attribute.

Similarly, some precedents in literature incorporate ergonomic parameters into the line balancing problems, in addition to the technological and managerial restrictions discussed so far. Indeed, Otto and Scholl (Otto and Scholl, 2011) proposed two ways to consider the ergonomic risk in the workstations of a line for the SALBP-1. The first one consists of adding constraints that limit the maximum allowed ergonomic risk; and the second proposal defines a new objective function that minimizes the number of workstations and the global ergonomic risk of the line using a weighting coefficient. In both proposals, they incorporated the ergonomic risk of an assembly line by means of three methods; the revised NIOSH (the National Institute for Occupational Safety and Health) equation and the job strain index; the OCRA (Occupational Repetitive Action) method; and the EAWS (European Assembly Worksheet) method, which was created for assembly production systems. In the same vein, other authors have also incorporated ergonomic parameters into line balancing problems. Bautista et al., $(2012,2013)$ used constraints to limit the maximum and minimum risk allowed at each workstation of the line within the TSALBP family of problems. Thus, the authors proposed a new family of problems called TSALBP_erg. Specifically these authors (Bautista et al., 2012, 2013) consider that ergonomic risk, within manufacturing 
environments, is given basically by the components related to both somatic and psychological comfort.

The psychological comfort refers to the set of mental conditions required by workers toper form their work. These conditions are autonomy, social support, acceptable workloads and a favorable work environment. There are several methods to evaluate this component of ergonomic risk, such as the COPSOQ (Copenhagen Psychosocial Questionnaire) that was adapted and validated in Spain with the name of ISTAS 21, the LEST method that was developed by the "Laboratoire d'Economie et Sociologie du Travail" and other methods with less reliability.

The somatic comfort concerns the set of physical demands to which a worker is exposed throughout the workday; physical demands that can potentially cause muscle contractions that compress nerve and vascular structures and induce chronic pain. In most cases, this pain is located in the upper extremities and back. There are several specific methods that analyze different risk factors to assess these types of ergonomic risk, such as postural loads, repetitive movements and manual handling.

- Postural loads: The workers may adopt inappropriate, asymmetric or awkward postures throughout the workday. These postures can cause certain stress to one or more anatomical regions. Some of these postural loads are hyper-extensions, hyper-flexions and hyper-rotations that may result in fatigue and musculoskeletal disorders over the long term. The methods found in the literature to analyze these types of ergonomic risk factors are the RULA (Rapid Upper Limb Assessment) (McAtamney and Corlett, 1993), the REBA (Rapid Entire Body Assessment) (Hignett and McAtamney, 2000) and the OWAS (Ovako Working Analysis System) (Karhu et al., 1977).

- Repetitive movements: the worker can perform several operations or activities involving effort and rapid or repetitive motion of small muscle groups. This set of repeated upperlimb movements may cause long term musculoskeletal injuries. To assess the ergonomic risk that involves this type of movement we use the OCRA Check List (Occupational Repetitive Action) (Colombini et al., 2002).

- Manual handling: Some tasks performed by workers involve the object lifting, movement, push, grip and transport that may be physically harmful. The NIOSH equation (National Institute for Occupational Safety and Health) (Waters et al., 1997) and the Tables from S.H. Snook and V. M. Ciriello (Snook and Ciriello, 1991) are methods to analyze this risk factor. 
Despite the large number of available methods to assess ergonomic risks, one of the major drawbacks found is the lack of unification of these methods. The specialization of each method into a single muscle disorder, complicates the assessment and granting of an ergonomic risk level given a job with all musculoskeletal disorders (TME) that are caused by postural loads, repetitive movements and manual handling. For this reason, we propose the following unified classification of the risk levels (Table 2).

Table 2: Classification of the level of risk by categories and actions to consider

\begin{tabular}{ccl}
\hline Level of risk & Category $(\chi)$ & \multicolumn{1}{c}{ Suggested action } \\
\hline Acceptable & 1 & $\begin{array}{l}\text { No action is required because there is no risk to the worker. } \\
\text { An analysis of the workstation is necessary. In the future, corrective actions for } \\
\text { its improvement are recommended. }\end{array}$ \\
High & 2 & $\begin{array}{l}\text { An analysis and improvement of the workstation and medical supervision are } \\
\text { immediately required. Regular checks are also recommended. } \\
\text { Immediate modification of the workstation is required because of the worker } \\
\text { presents serious illness }\end{array}$ \\
\hline
\end{tabular}

The above classification (Table 2) allows us to determine the risk level of tasks in regard with the somatic comfort, considering postural loads, repetitive movements and manual handling simultaneously. In this way, we can obtain an only risk value for all the set of tasks assigned to a workstation, from the ergonomic levels defined by the RULA, OCRA and NIOSH methods(at our discretion), i.e., we can determine the overall risk level to which the workers will be subjected throughout their workday.

On the other hand, and taking into consideration the unified classification for the ergonomic risk associated to the somatic factors (postural loads, repetitive movements and manual handling), we propose a new approach to incorporate into the TSALBP these ergonomic factors that may be harmful to the health of workers. Specifically, our objective is to improve the researches published by Bautista and Pereria (2007) and Chica et al., (2010) that, respectively, focus on (1) studying the TSALBP-mminimizing the number of stations (limiting the cycle time and the linear area) for a single instance (\# 1) which corresponds to a production mix with an identical daily demand for all types of engines; (2) studying the biobjective problem that minimizes the number of workstations of the line $(m)$ and the maximum linear area required by the stations $(A)(T S A L B P-m / A)$.

As a result, the main differences between the present paper and the researches by Bautista and Pereria (2007) and Chica et al. (2010) are the following:

- In this research, we use a case study that consists of nine demand plans. These demand plans correspond with the daily production of 270 engines, which are divided into nine types with 
different characteristics and therefore different use of resources and different value for the temporal and ergonomic attributes. Each plan represents a different production mix.

- We propose nine different configurations for the assembly line of engines.

- We balance the assembly line with a new methodology. Specifically, we minimize the difference between the real and ideal or average values in regard with the temporal, spatial and ergonomic attributes ( $T^{\text {med }}, A^{\text {med }}$ and $\left.R^{\text {med }}\right)$.

- The final solution is obtained by phases. The solutions that do not satisfy the nine demand plans and the maximum admissible values for attributes are rejected at each phase. In this way, in the last phase, the most robust configuration, i.e., the solution that satisfies the nine demand plans with fewer changes at workstations when the mix production varies, is obtained.

- In this work, not only the managerial and technological characteristics are fulfilled. Now, we guarantee that operators are exposed to acceptable levels of ergonomic risks.

In view of above, the present paper is organized as follows: In section 2, the new approach to solve line balancing problem is explained. This section presents the starting considerations for this approach, the parameters and variables used and the proposed mathematical models. Section 3 describes the making-decision methodology proposed to select the configurations more closely related and robust configurations for the mixed-model assembly line. Besides, the criterions used to reject or select the configurations are also defined. Section 4describes the computational experiment carried out and linked to a case study of Nissan. Once the experiment is explained, the obtained results are analyzed, taking into account the attributes considered in the new approach and the proposed methodology to decide what configurations are the most appropriate for the demand plan variations and the maximum allowable levels of ergonomic risk. Finally, Section 5 presents the conclusion of the paper.

\section{Models for assembly line balancing by attributes}

At this point of paper, the new approaches for balancing assembly lines are presented from the mathematical models of TSALBP family and the unification of methods for assessing somatic risk factors. Specifically, the nomenclature used in this research, as well as new balancing functions that consider aspects of management, technology and ergonomics, are defined after a series of preliminaries. Finally, new models for line balancing are formulated, summarizing the contributions made at the end. 


\subsection{Preliminaries}

Given the set $J$ of elemental tasks and the set $K$ of workstations, the assembly line balancing problem consists of establishing task assignments $j \in J$ to these workstations $k \in K$ in order to satisfy the set of technological, managerial and ergonomic constraints.

In our approach, we distinguish the following problem aspects:

1. The objective of the line-balancing problem.

2. The attributes associated with each objective of the balancing problem.

3. The line balancing characterization.

4. Types of restrictions and functions involved in the problem.

In the first place, the balancing problem can have as objective: (1) the processing times of tasks at workstations; (2) the space given to the workers to perform their work; and (3) the risk of injury from the tasks assigned to the workers.

Each one of these study objects may be associated with a set of attributes. We can find temporal attributes, such as the processing time of a task, the cycle time $(c)$, the workload time of a workstation or the discrepancy between this time and the ideal value for the workload time. Likewise, the area required by each task, the area available for a workstation, the area linked to the workload assigned to a workstation and the minimum number of work stations are spatial attributes. And we can also find some attributes associated with the risk of injury such as the risk category of a task, the processing time and the ergonomic risk of one task or workstation.

Based on the above, we can characterize the assembly line balancing by three ways: (1) by means of imposing restrictions to the attributes (temporal, spatial an ergonomic); (2) by the optimization of one or more attributes; and (3) through the simultaneos use of constraints and optimization criteria.

Regarding the type of restrictions, these are assignments, incompatibilities, groups, precedence rules and limitations of the reference value of attributes. Likewise, the proposed objective functions will serve to reach a minimum compatible value of one or more attributes linked to the time, space or risk (compatibility problem) or to obtain solutions whose attributes will be adjusted to their best possible reference values (adjustment problems). Finally, we must consider the automotive sector from the $O E C D$ (Organization for Economic Co-operation and Development) (the geographical framework of our study) presents some features that limit the usefulness of some models of line balancing. Specifically, the models must consider the following: (1) the automotive lines are oriented to mixed-products (i.e., 
engines for different vehicles types) ; (2) the partial and global product demands vary frequently over the year (a few times each month); (3) the workforce contracting regimes are not very flexibles in the $O E C D$, that which is convenient for maintaining the loyalty of workers to the company; and (4)the product demand variation produces new task assignments to the workstations and this supposes that the worker training can last weeks until reaching the continuous operation.

For all the above reasons, we propose balancing models with a fixed workforce and considering the three ways to characterize the problem. Therefore, we consider a fixed number of workstations, $m$, and different demand plans with different partial demand of product types.

\subsection{Nomenclature}

Next, before defining new functions for balancing mixed-model assembly lines and formulate new mathematical models, the sets of parameters and variables used by the balancing models by attributes are presented.

Parameters

$$
\begin{array}{ll}
\hline J & \text { Set of elemental tasks }(j=1 \ldots . .|J|) . \\
K & \text { Set of workstations }(k=1 \ldots . .|K|) . \\
m & \text { Number of workstations, } m=|K|, \text { that is known and fixed. } \\
\Phi & \text { Set of ergonomic risk factors }(\phi=1, \ldots,|\Phi|) . \\
t_{j} & \text { Processing time of an elemental task } j \in J \text { (at normal work pace or activity level). } \\
a_{j} & \text { Area or space (linear) required by the task } j \in J . \\
\chi_{\phi, j} & \text { Ergonomic risk category associated to the task } j \in J \text { regarding the risk factor }
\end{array}
$$

$R_{\phi, j} \quad$ Ergonomic risk associated to the task $j \in J$ regarding the risk factor

$$
\phi \in \Phi \cdot R_{\phi, j}=\chi_{\phi, j} \cdot t_{j}
$$

$P_{j} \quad$ Set of tasks that precede the task $j \in J$.

$T_{k}^{\max } \quad$ Maximum processing time (at normal activity level) given to the workstation $k \in K$.

$T^{\text {med }} \quad$ Average processing time (at normal activity level) of each workstation while manufacturing a product unit. That is: $T^{m e d}=\frac{1}{|K|} \sum_{j=1}^{|J|} t_{j}$. 
$A_{k}^{\max } \quad$ Maximum area (linear) available at workstation $k \in K$ to perform the tasks.

$A^{\text {med }} \quad$ Average area (linear) corresponding to each workstation to perform the task. That is: $A^{\text {med }}=\frac{1}{|K|} \sum_{j=1}^{|J|} a_{j}$.

$R_{\phi, k}^{\max } \quad$ Maximum ergonomic risk allowed at workstation $k \in K$ according to the ergonomic risk factor $\phi \in \Phi$.

$R_{\phi}^{\text {med }} \quad$ Average ergonomic risk assumed by each workstation regarding the risk factor $\phi \in \Phi$. That is: $R_{\phi}^{\text {med }}=\frac{1}{|K|} \sum_{j=1}^{|J|} R_{\phi, j}, \forall \phi \in \Phi$.

Variables

$x_{j, k} \quad$ Binary variable equal to 1 if the task $j \in J$ is assigned to the workstation $k \in K$ and 0 otherwise.

$S_{k} \quad$ Workload of workstation $K$. The set of tasks assigned to the workstation $k \in K$ : $S_{k}=\left\{j \in J: x_{j, k}=1\right\}$.

$T\left(S_{k}\right) \quad$ Processing time required (at normal activity) to perform the workload $S_{k}$ : $T\left(S_{k}\right)=\sum_{j \in S_{k}} t_{j}$

$A\left(S_{k}\right) \quad$ Area (linear) required by the workload $S_{k}: A\left(S_{k}\right)=\sum_{j \in S_{k}} a_{j}$.

$R_{\phi}\left(S_{k}\right) \quad$ Ergonomic risk for the factor $\phi \in \Phi$ associated to the workload $S_{k}: R_{\phi}\left(S_{k}\right)=\sum_{j \in S_{k}} R_{\phi, j}$.

$\delta_{k}^{+}(T) \quad$ Over-time (at normal work pace) required at workstation $k \in K$ with respect to the average value. That is: $\delta_{k}^{+}(T)=\left[T\left(S_{k}\right)-T^{\text {med }}\right]^{+}$, with $[x]^{+}=\max \{0, x\}$.

$\delta_{k}^{-}(T) \quad$ Defect of processing time required by the workstation $k \in K$ (at normal activity) with respect to the average value. That is: $\delta_{k}^{-}(T)=\left[T^{\text {med }}-T\left(S_{k}\right)\right]^{+}$, with $[x]^{+}=\max \{0, x\}$.

$\delta_{k}^{+}(A) \quad$ Over-area (linear) needed at workstation $k \in K$ with respect to average area: $\delta_{k}^{+}(A)=\left[A\left(S_{k}\right)-A^{\text {med }}\right]^{+}$.

$\delta_{k}^{-}(A) \quad$ Area defect (linear) needed at workstation $k \in K$ with respect to its 
average: $\delta_{k}^{-}(A)=\left[A^{\text {med }}-A\left(S_{k}\right)\right]^{+}$.

$\delta_{\phi, k}^{+}(R) \quad$ Over-ergonomic risk at workstation $k \in K$ depending on the factor $\phi \in \Phi$ with respect to its average $\delta_{\phi, k}^{+}(R)=\left[R_{\phi}\left(S_{k}\right)-R_{\phi}^{\text {med }}\right]^{+}$.

$\delta_{\phi, k}^{-}(R)$ Ergonomic risk defect at workstation $k \in K$ depending on the factor $\phi \in \Phi$ with respect to its average $\delta_{\phi, k}^{-}(R)=\left[R_{\phi}^{\text {med }}-R_{\phi}\left(S_{k}\right)\right]^{+}$.

$T_{k}^{\max } \quad$ Maximum processing time (at normal activity level) given to the workstation $k \in K$.

$A_{k}^{\max } \quad$ Maximum area (linear) available at workstation $k \in K$ to perform the tasks.

$R_{\phi, k}^{\max } \quad$ Maximum ergonomic risk allowed at workstation $k \in K$ according to the ergonomic risk factor $\phi \in \Phi$.

Note that parameters $T_{k}^{\max }, A_{k}^{\max }$ and $R_{\phi, k}^{\max }$ also can be considered as variables.

In this research and specifically in the proposed models, areas are defined by the proxy variable "linear area", which is measured in units of length. We assume that the working space on both sides of the assembly line (i.e., where the workers move about and where components are stored) has a homogeneous width along the line, and it is enough for a comfortable work. In consequence, only the length of the workstations should be taken into account in the optimization process.

On the other hand, the ergonomic risk is measured in ergo-seconds (e-s). An ergo-second is the time unit, measured in seconds, used to assess the ergonomic risk of a task, with a processing time of 1 second at normal work pace, bearing a risk category of 1 . Thus, this scale measures the time spent by workers to perform a task (at normal pace) taking into account the level of the ergonomic risk to which they are exposed.

From all of these parameters and variables we are able to formulate the proposed models.

\subsection{Balancing functions}

Concerning the characterization through objective functions, there are many objective functions for balancing problems, in the literature, which consider several attributes (see Battaïa and Dolgui, 2013). In our case, we consider three attribute types: temporal, spatial and ergonomic; and two function types: compatibility and adjustment functions. 


\section{Elemental compatibility functions:}

This type of function limits the attribute values. Therefore, we have three different functions in regard with the temporal, spatial and ergonomic attributes.

First, we have the temporal limitation function:

$$
c=\max _{k \in K}\left(T_{k}^{\max }\right)
$$

Where $c$ is the cycle time (to minimize) and $T_{k}^{\max }$ is a real variable that represents the processing time (at normal activity) needed by the workstation $k \in K$ to perform its workload or assigned tasks $\left(S_{k}\right)$. For the function (1), $A_{k}(\forall k)$ and $R_{\phi, k}(\forall k, \forall \phi)$ are considered parameters.

Secondly, considering the spatial attribute, we define function for the linear area limitation at workstations:

$$
A=\max _{k \in K}\left(A_{k}^{\max }\right)
$$

Where $A$ is the linear area (to minimize) given to each workstation and $A_{k}^{\max }$ is the area required by the workload $S_{k}$. For the function (2), $T_{k}(\forall k)$ and $R_{\phi, k}(\forall k, \forall \phi)$ are considered parameters.

Finally, we have the function for the ergonomic risk:

$$
R=\max _{k \in K}\left\{\max _{\phi \in \Phi}\left(R_{\phi, k}\right)\right\}
$$

Where $R$ is the maximum ergonomic risk (to minimize) allowed to each workstation $(k \in K)$ for any risk factor $\phi \in \Phi$, and $R_{\phi, k}$ is the ergonomic risk that generates the workload $S_{k}$ regarding the factor $\phi$. For function (3), $T_{k}(\forall k)$ and $A_{k}(\forall k)$ are parameters.

\section{Elemental adjustment functions:}

The elemental adjustment functions focus on reducing the discrepancies or distances between the real values for temporal, spatial and ergonomic attributes given by the assignments of task to workstations and the ideal reference values fixed by the attributes.

For these types of functions, $T_{k}^{\max }, A_{k}^{\max }(\forall k)$ and $R_{\phi, k}^{\max }(\forall k, \forall \phi)$ are parameters with fixed and known values. 
Thus, taking in mind the different measures for distance, rectangular, Euclidean and quadratic, we propose the following adjustment functions:

a) Functions with temporal attributes:

$$
\begin{gathered}
\Delta_{R}(T)=\sum_{k=1}^{|K|}\left[\delta_{k}^{+}(T)+\delta_{k}^{-}(T)\right] \\
\Delta_{E}(T)=\sum_{k=1}^{|K|} \sqrt{\delta_{k}^{+}(T)^{2}+\delta_{k}^{-}(T)^{2}} \\
\Delta_{Q}(T)=\sum_{k=1}^{|K|}\left[\delta_{k}^{+}(T)^{2}+\delta_{k}^{-}(T)^{2}\right]
\end{gathered}
$$

Where $\Delta_{R}(T), \Delta_{E}(T)$ and $\Delta_{Q}(T)$ are the overall discrepancies of the workload times of workstations with regard to the average value, measured according to rectangular, Euclidean and quadratic distances, respectively.

b) Functions with spatial attributes:

$$
\begin{gathered}
\Delta_{R}(A)=\sum_{k=1}^{|K|}\left[\delta_{k}^{+}(A)+\delta_{k}^{-}(A)\right] \\
\Delta_{E}(A)=\sum_{k=1}^{|K|} \sqrt{\delta_{k}^{+}(A)^{2}+\delta_{k}^{-}(A)^{2}} \\
\Delta_{Q}(A)=\sum_{k=1}^{|K|}\left[\delta_{k}^{+}(A)^{2}+\delta_{k}^{-}(A)^{2}\right]
\end{gathered}
$$

Where $\Delta_{R}(A), \Delta_{E}(A)$ and $\Delta_{Q}(A)$ are the overall discrepancies (rectangular, Euclidean and quadratic) between areas required by the workload, $S_{k}$, at workstations ( $k \in K$ ) and the average of the areas required by tasks at the set of workstations.

c) Functions with ergonomic risk attributes:

$$
\begin{aligned}
\Delta_{R}(R) & =\sum_{k=1}^{|K|} \sum_{\phi=1}^{|\Phi|}\left[\delta_{\phi, k}^{+}(R)+\delta_{\phi, k}^{-}(R)\right] \\
\Delta_{E}(R) & =\sum_{k=1}^{|K|} \sum_{\phi=1}^{|\Phi|} \sqrt{\delta_{\phi, k}^{+}(R)^{2}+\delta_{\phi, k}^{-}(R)^{2}}
\end{aligned}
$$




$$
\Delta_{Q}(R)=\sum_{k=1}^{|K|} \sum_{\phi=1}^{|\Phi|}\left[\delta_{\phi, k}^{+}(R)^{2}+\delta_{\phi, k}^{-}(R)^{2}\right]
$$

Where $\Delta_{R}(R), \Delta_{E}(R)$ and $\Delta_{Q}(R)$ are the addition discrepancies (rectangular, Euclidean and quadratic) of the ergonomic risks regarding the average values for each risk factor $\phi \in \Phi$. In short, all the defined set of functions by attributes are the following:

\begin{tabular}{|c|c|c|}
\hline Type & Nomenclature & Objetive \\
\hline \multirow{3}{*}{ 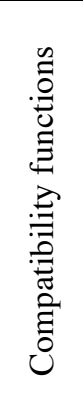 } & $c$ & $\begin{array}{l}\text { Maximization of the production rate of the line, with a linear area limited, } A \text {, } \\
\text { and ergonomic risk limited, } R \text {, per workstation. That is equivalent to } \\
\text { minimizing the cycle time, } c \text {. }\end{array}$ \\
\hline & $A$ & $\begin{array}{l}\text { Minimization of the space required by each workstation of the line, limiting } \\
\text { the cycle time, } c \text {, and the ergonomic risk, } R \text {. }\end{array}$ \\
\hline & $R$ & $\begin{array}{l}\text { Minimization of the risk of injuries for the workers of the line, limiting the } \\
\text { cycle time, } c \text {, and the linear area per station, } A \text {. }\end{array}$ \\
\hline \multirow{3}{*}{ 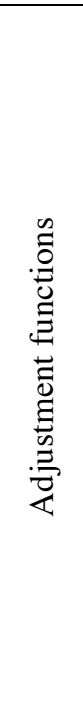 } & $\Delta_{R}(T), \Delta_{E}(T), \Delta_{Q}(T)$ & $\begin{array}{l}\text { Minimization of the discrepancy between the cycle time needed to carry out } \\
\text { the tasks at each workstation and the average cycle time assigned to each } \\
\text { station, limiting the area, } A \text {, and the risk, } R \text {. This discrepancy can be } \\
\text { measured by rectangular, Euclidean and quadratic distance. }\end{array}$ \\
\hline & $\Delta_{R}(A), \Delta_{E}(A), \Delta_{Q}(A)$ & $\begin{array}{l}\text { Minimization of the discrepancy between the linear area required by the } \\
\text { operations assigned to each station and the average area assigned, limiting } \\
\text { the cycle time, } c \text {, and the ergonomic risk, } R \text {. The discrepancy can be } \\
\text { measured through rectangular, Euclidean and quadratic distance. }\end{array}$ \\
\hline & $\Delta_{R}(R), \Delta_{E}(R), \Delta_{Q}(R)$ & $\begin{array}{l}\text { Minimization of the rectangular, Euclidean or quadratic distance between } \\
\text { the ergonomic risk associated to the tasks assigned to workstations and the } \\
\text { average ergonomic risk per station. In this case, the limiting attributes are } \\
\text { the cycle time, } c \text {, and the area allowed per station, } A \text {. }\end{array}$ \\
\hline
\end{tabular}

\subsection{Feasibility Model}

The first model we propose is based on the characterization of line balancing using constraints associated with the problem's attributes. In this case, we consider the three types of attributes considered above: temporal, spatial and risk of injury.

Therefore, given an assignment of tasks to the workstations by means of the binary parameters $x_{j, k}^{\circ} \in\{0,1\}(\forall j \in J, \forall k \in K)$, the model will check if this assignment is feasible, 
i.e., whether all the constraints, such as precedence tasks and maximum values for the attributes, are satisfied. The $M_{-} A A L B \_\varnothing$ model is the following:

$M_{-} A A L B_{-} \varnothing$ model:

$x_{j, k}=x_{j, k}^{\circ}$

$(j=1, \ldots,|J|)(k=1, \ldots,|K|)$

$\sum_{k=1}^{|K|} x_{j, k}=1$

$(j=1 \ldots . .|J|)$

$\sum_{j=1}^{|J|} x_{j, k} \geq 1$

$(k=1 \ldots . .|K|)$

$\sum_{k=1}^{|K|} k\left(x_{i, k}-x_{j, k}\right) \leq 0$

$i \in P_{j}, j=1, \ldots|J|$

$\sum_{j=1}^{|J|} t_{j} \cdot x_{j, k} \leq T_{k}^{\max }$

$(k=1 \ldots . .|K|)$

$\sum_{j=1}^{|J|} a_{j} \cdot x_{j, k} \leq A_{k}^{\max }$

$(k=1 \ldots . .|K|)$

$\sum_{j=1}^{|J|} R_{\phi, j} \cdot x_{j, k} \leq R_{\phi, k}^{\max }$

$(k=1, \ldots,|K|)(\phi=1, . . . \Phi \mid)$

$\sum_{j=1}^{|J|} t_{j} \cdot x_{j, k}-\delta_{k}^{+}(T)+\delta_{k}^{-}(T)=T^{m e d}$

$(k=1 \ldots . .|K|)$

$\sum_{j=1}^{|J|} a_{j} \cdot x_{j, k}-\delta_{k}^{+}(A)+\delta_{k}^{-}(A)=A^{m e d}$

$(k=1 \ldots . .|K|)$

$\sum_{j=1}^{|J|} R_{\phi, j} \cdot x_{j, k}-\delta_{\phi, k}^{+}(R)+\delta_{\phi, k}^{-}(R)=R_{\phi}^{m e d}$

$(k=1, \ldots,|K|)(\phi=1, . .|\Phi|)$

$\delta_{k}^{+}(T), \delta_{k}^{+}(A), \delta_{k}^{-}(T), \delta_{k}^{-}(A) \geq 0$

$(k=1 \ldots . .|K|)$

$\delta_{\phi, k}^{+}(R), \delta_{\phi, k}^{-}(R) \geq 0$

$(k=1, \ldots,|K|)(\phi=1, . .|\Phi|)$

In the $M_{-} A A L B \_\varnothing$ model the equality (13) establishes the assignments of tasks to workstations in order to prove whether these assignments are feasible. Constraint (14) indicates that each task can only be assigned to one workstation. Constraint (15) forces any workstation, $|K|$, to not be empty. Constraint (16) corresponds to the precedence task bindings. Constraints (17), (18) and (19) impose the maximum limitation of the workload 
time, area required by the workload and ergonomic risk generated by the workload assigned to each workstation. Constraints (20), (21) and (22) define the temporary, spatial and ergonomic risk discrepancies, both positive and negative, between the average and real values for each workstation. Finally, the constraints (23) and (24) establish the non-negativity of the variables.

\subsection{Optimization Models}

The second proposal consists of the optimization of one or more attributes. From the feasibility $M_{-} A A L B_{-} \varnothing$ model and the balancing functions defined above, we can formulate a family of optimization models for assembly line balancing, where the number of workstations is a parameter previously fixed and where the objective is to minimize the cycle time, the required area or/and the ergonomic risk or the discrepancies between the real and the ideal values for the said attributes.

To do this, we must first define the following function sets:

$$
\begin{aligned}
& \mathfrak{\Im}_{T}=\left\{c, \Delta_{R}(T), \Delta_{E}(T), \Delta_{Q}(T)\right\} \\
& \mathfrak{\Im}_{A}=\left\{A, \Delta_{R}(A), \Delta_{E}(A), \Delta_{Q}(A)\right\} \\
& \mathfrak{I}_{R}=\left\{R, \Delta_{R}(R), \Delta_{E}(R), \Delta_{Q}(R)\right\}
\end{aligned}
$$

a) Mono-objective models:

$$
\left\{\begin{array}{l}
\operatorname{Minf} f_{T} \in \mathfrak{I}_{T} \\
\operatorname{Minf} f_{A} \in \mathfrak{I}_{A} \\
\operatorname{Minf}_{R} \in \mathfrak{I}_{R}
\end{array}\right\}
$$

Subject to: (14)-(24) from $M_{-} A A L B_{-} \varnothing$

$$
x_{j, k} \in\{0,1\} \quad(j=1, \ldots,|J|)(k=1, \ldots,|K|)
$$

b) Bi-objective models:

$$
\left\{\begin{array}{c}
{\left[\left(\operatorname{Minf}_{T} \in \mathfrak{\Im}_{T}\right) \wedge\left(\operatorname{Minf} f_{A} \in \mathfrak{I}_{A}\right)\right] \vee} \\
{\left[\left(\operatorname{Minf}_{T} \in \mathfrak{\Im}_{T}\right) \wedge\left(\operatorname{Minf} f_{R} \in \mathfrak{I}_{R}\right)\right] \vee} \\
{\left[\left(\operatorname{Minf} f_{A} \in \mathfrak{I}_{A}\right) \wedge\left(\operatorname{Minf} f_{R} \in \mathfrak{I}_{R}\right)\right]}
\end{array}\right\}
$$

Subject to: (14)-(24) from $M_{-} A A L B \_\varnothing$ 


$$
x_{j, k} \in\{0,1\} \quad(j=1, \ldots,|J|)(k=1, \ldots,|K|)
$$

c) Tri-objective model:

$$
\left(\operatorname{Minf}_{T} \in \mathfrak{I}_{T}\right) \wedge\left(\operatorname{Minf}_{A} \in \mathfrak{I}_{A}\right) \wedge\left(\operatorname{Minf}_{R} \in \mathfrak{I}_{R}\right)
$$

Subject to: (14)-(24) from $M_{-} A A L B \_\varnothing$

$$
x_{j, k} \in\{0,1\} \quad(j=1, \ldots,|J|)(k=1, \ldots,|K|)
$$

d) Weighted attribute models:

For these models the following weighted attribute functions are valid:

$$
\begin{aligned}
& \Gamma(T, A, R)=\mu_{T} \cdot c+\mu_{A} \cdot A+\mu_{R} \cdot R \\
& \Delta_{R}(T, A, R)=\mu_{T} \cdot \Delta_{R}(T)+\mu_{A} \cdot \Delta_{R}(A)+\mu_{R} \cdot \Delta_{R}(R) \\
& \Delta_{E}(T, A, R)=\mu_{T} \cdot \Delta_{E}(T)+\mu_{A} \cdot \Delta_{E}(A)+\mu_{R} \cdot \Delta_{E}(R) \\
& \Delta_{Q}(T, A, R)=\mu_{T} \cdot \Delta_{Q}(T)+\mu_{A} \cdot \Delta_{Q}(A)+\mu_{R} \cdot \Delta_{Q}(R)
\end{aligned}
$$

Where $\mu_{T}, \mu_{A}, \mu_{R}$ are parameters, measured in $\operatorname{seconds}^{-1}\left(\mu_{T}\right)$, centimeters ${ }^{-1}\left(\mu_{A}\right)$ and ergoseconds $^{-1}\left(\mu_{R}\right)$, and multipliers of the attributes of workload time, area and ergonomic risk respectively. These parameters must satisfy:

$\mu_{T} T^{\text {med }}+\mu_{A} A^{\text {med }}+\mu_{R} R^{\text {med }}=1$

$\mu_{T}, \mu_{A}, \mu_{R} \geq 0$

And where $R^{\text {med }}=\frac{1}{|\Phi|} \sum_{\phi=1}^{|\Phi|} R_{\phi}^{\text {med }}$.

In such conditions, we can define the following model with weighted attributes:

$$
\operatorname{Min} f \in\left\{\Gamma(T, A, R), \Delta_{R}(T, A, R), \Delta_{E}(T, A, R), \Delta_{Q}(T, A, R)\right\}
$$

Subject to: (14)-(24) from $M_{-} A A L B \_\varnothing$

$$
x_{j, k} \in\{0,1\} \quad(j=1, \ldots,|J|)(k=1, \ldots,|K|)
$$

The latter model will be the base of our case study, specifically with the use of the $\Delta_{R}(T, A, R)$ weighted function. 


\section{Decision-making by incorporating affinity and robustness}

\subsection{Previous definitions}

\section{Similarity degree between solutions}

Given two configurations $\zeta_{0}=n$ and $\zeta_{0}=n^{\prime}$, that have been obtained by weighted model, $M_{-} A A L B \_\Delta_{R}(T, A, R)$, and whose workstations' workloads are $\vec{S}_{\zeta_{0}}=\left(S_{1, \zeta_{0}}, \ldots, S_{m, \zeta_{0}}\right)$ with $\zeta_{0} \in\left\{n, n^{\prime}\right\}$, we define the following affinity index for each workstation $(\forall k \in K)$ :

$$
A\left(S_{k, n}, S_{k, n^{\prime}}\right)=\frac{2\left|S_{k, n} \cap S_{k, n^{\prime}}\right|}{\left|S_{k, n}\right|+\left|S_{k, n^{\prime}}\right|} \quad \forall k=1, \ldots, m
$$

Then, considering all the set of workstations, we can denote the affinity index between the configurations $\zeta_{0}=1$ and $\zeta_{0}=2$ as follows:

$$
A\left(\vec{S}_{n}, \vec{S}_{n^{\prime}}\right)=\frac{\sum_{k=1}^{m} A\left(S_{k, n}, S_{k, n^{\prime}}\right)}{m}
$$

As a result, the similarity between two configurations, $\zeta_{0}=1$ and $\zeta_{0}=2$, will be complete if the index $A\left(\vec{S}_{n}, \vec{S}_{n^{\prime}}\right)$ adopts the value 1 .

\section{Robustness of a solution}

To measure the "robustness" degree of a line configuration according to the line's attributes, we focus on two indicator types:

1. Maximum excesses, regarding the average values of workload time, required area and ergonomic risk, obtained when the demand plans vary ( $g_{1}$ index type).

2. Overall excesses, regarding the average values of workload time, required area and ergonomic risk, produced by line and set of demand plans, E, ( $g_{2}$ index type).

Before defining these robustness indices, we state the following parameters:

$S_{k, \zeta_{0}} \quad$ Workload (set of tasks) assigned to the workstation $k \in K$, and which corresponds to the $\zeta_{0}$ configuration.

$t\left(S_{k, \xi_{0}}, \# \varepsilon\right) \quad$ Workload time corresponding to the workload, $S_{k, \xi_{0}}$, when the processing times of tasks linked to the demand plan $\# \varepsilon \in \mathrm{E}$ are considered. 
$a\left(S_{k, \zeta_{0}}, \# \varepsilon\right) \quad$ Linear area corresponding to the workload, $S_{k, \zeta_{0}}$, when the areas required by tasks linked to the demand plan \# $\varepsilon \in \mathrm{E}$ are used.

$R_{\phi}\left(S_{k, \zeta_{0}}, \# \varepsilon\right)$ Ergonomic risk corresponding to the workload $S_{k, \xi_{0}}$ for the risk factor $\phi \in \Phi$ when the ergonomic risks of tasks associated with the demand plan $\# \varepsilon \in \mathrm{E}$, are used.

$\bar{T}(\# \varepsilon) \quad$ Average processing time (at normal activity), by workstation, to perform a product unit when the task processing times linked to the demand plan $\# \varepsilon \in$ Eare used.

$\bar{A}(\# \varepsilon) \quad$ Average linear area allowed at each workstation to process a product unit when the areas required by the tasks from the demand plan $\# \varepsilon \in \mathrm{E}$ are used. In our case, $\bar{A}(\# \varepsilon)=c t e \forall \# \varepsilon \in \mathrm{E}$.

$\bar{R}_{\phi}(\# \varepsilon) \quad$ Average ergonomic risk, for the risk factor $\phi \in \Phi$ associated to each workstation, given by the ergonomic risk of the demand plan $\# \varepsilon \in \mathrm{E}$.

Consequently, given the set of workstations $k(k=1, \ldots, m=|K|)$, the set E of demand plans $(\# \varepsilon=1, \ldots,|\mathrm{E}|)$ and the line configuration that corresponds to the best solution of the balancing line obtained from the demand plan, we can define the following non-resilience indices.

a) Proportion of the maximum excesses of the attributes, such as processing time, required area and ergonomic risk, with regard to their average values.

$$
\begin{gathered}
g_{1}\left(\zeta_{0}, T\right)=\max _{k \in K} \max _{\# \varepsilon \in E}\left\{\frac{1}{\bar{T}(\# \varepsilon)}\left[t\left(S_{k, \zeta_{0}}, \# \varepsilon\right)-\bar{T}(\# \varepsilon)\right]^{+}\right\} \\
g_{1}\left(\zeta_{0}, A\right)=\max _{k \in K} \max _{\# \varepsilon \in E}\left\{\frac{1}{\bar{A}(\# \varepsilon)}\left[a\left(S_{k, \zeta_{0}}, \# \varepsilon\right)-\bar{A}(\# \varepsilon)\right]^{+}\right\} \\
g_{1}\left(\zeta_{0}, R_{\phi}\right)=\max _{k \in K} \max _{\# \varepsilon \in \mathrm{E}}\left\{\frac{1}{\overline{R_{\phi}}(\# \varepsilon)}\left[R_{\phi}\left(S_{k, \zeta_{0}}, \# \varepsilon\right)-\overline{R_{\phi}}(\# \varepsilon)\right]^{+}\right\}
\end{gathered}
$$

b) Proportion of the overall excesses of the attributes, such as processing time, required area and ergonomic risk, with respect to their average values. 


$$
\begin{gathered}
g_{2}\left(\zeta_{0}, T\right)=\frac{1}{m \cdot|\mathrm{E}|} \cdot \sum_{k \in K} \sum_{\# \varepsilon \in \mathrm{E}}\left\{\frac{1}{\bar{T}(\# \varepsilon)}\left[t\left(S_{k, \zeta_{0}}, \# \varepsilon\right)-\bar{T}(\# \varepsilon)\right]^{+}\right\} \\
g_{2}\left(\zeta_{0}, A\right)=\frac{1}{m \cdot|\mathrm{E}|} \cdot \sum_{k \in K} \sum_{\# \in \in \mathrm{E}}\left\{\frac{1}{\bar{A}(\# \varepsilon)}\left[a\left(S_{k, \zeta_{0}}, \# \varepsilon\right)-\bar{A}(\# \varepsilon)\right]^{+}\right\} \\
g_{2}\left(\zeta_{0}, R_{\phi}\right)=\frac{1}{m \cdot|\mathrm{E}|} \cdot \sum_{k \in K} \sum_{\# \varepsilon \in E}\left\{\frac{1}{\overline{R_{\phi}}(\# \varepsilon)}\left[R_{\phi}\left(S_{k, \zeta_{0}}, \# \varepsilon\right)-\overline{R_{\phi}}(\# \varepsilon)\right]^{+}\right\}
\end{gathered}
$$

Alternatively, the non-robustness indices are also valid if we use the maximum values for the attributes. That is:

a) Proportion of maximum excesses of the attributes (processing time, required area and ergonomic risk) concerning their maximum allowed values.

$$
\begin{aligned}
& g_{1}^{\max }\left(\zeta_{0}, T\right)=\max _{k \in K} \max _{\# \varepsilon \in}\left\{\frac{1}{T_{k}^{\max }}\left[t\left(S_{k, \zeta_{0}}, \# \varepsilon\right)-\bar{T}(\# \varepsilon)\right]^{+}\right\} \\
& g_{1}^{\max }\left(\zeta_{0}, A\right)=\max _{k \in K} \max _{\# \varepsilon \in E}\left\{\frac{1}{A_{k}^{\max }}\left[a\left(S_{k, \zeta_{0}}, \# \varepsilon\right)-\bar{A}(\# \varepsilon)\right]^{+}\right\} \\
& g_{1}^{\max }\left(\zeta_{0}, R_{\phi}\right)=\max _{k \in K} \max _{\# \varepsilon \in}\left\{\frac{1}{R_{\phi, k}^{\max }}\left[R_{\phi}\left(S_{k, \zeta_{0}}, \# \varepsilon\right)-\overline{R_{\phi}}(\# \varepsilon)\right]^{+}\right\}
\end{aligned}
$$

b) Proportion of the overall excesses of the attributes (processing time, required area and ergonomic risk) with regard to their maximum allowed values.

$$
\begin{aligned}
& g_{2}^{\max }\left(\zeta_{0}, T\right)=\frac{1}{m \cdot|\mathrm{E}|} \cdot \sum_{k \in K} \sum_{\# \in \in \mathrm{E}}\left\{\frac{1}{T_{k}^{\max }}\left[t\left(S_{k, \zeta_{0}}, \# \varepsilon\right)-\bar{T}(\# \varepsilon)\right]^{+}\right\} \\
& g_{2}^{\max }\left(\zeta_{0}, A\right)=\frac{1}{m \cdot|\mathrm{E}|} \cdot \sum_{k \in K} \sum_{\# \in \in \mathrm{E}}\left\{\frac{1}{A_{k}^{\max }}\left[a\left(S_{k, \zeta_{0}}, \# \varepsilon\right)-\bar{A}(\# \varepsilon)\right]^{+}\right\} \\
& g_{2}^{\max }\left(\zeta_{0}, R_{\phi}\right)=\frac{1}{m \cdot|\mathrm{E}|} \cdot \sum_{k \in K} \sum_{\# \varepsilon \in \mathrm{E}}\left\{\frac{1}{R_{\phi, k}^{\max }}\left[R_{\phi}\left(S_{k, \zeta_{0}}, \# \varepsilon\right)-\overline{R_{\phi}}(\# \varepsilon)\right]^{+}\right\}
\end{aligned}
$$




\subsection{Decision-making process}

To select the most appropriate configuration for a set of different demand plans, we define the following decision-making methodology, which is structured in five stages (Figure 1).

Step 1. Data collection: focusing on scenarios of demand plans, their processing times of operations, required areas, the risk category of the analyzed operations, as well as the boundaries of time, spatial and risk attributes. Collection and analysis of demand plans; determination of the processing times, required areas, and categories of risk of operations; determination of the limits of temporal, spatial and risk attributes.

Step 2.Line configuration's search: given a value range for the maximum ergonomic risk $R^{\max }$, and the number of workstations, $m$, the line configurations $\left(S_{k}, \ldots, \forall k \in K\right)$ that satisfy the demand plans are searched.

Step 3.Selection of dominant line configurations: from the set of configurations previously found, we select those configurations that: (1) are valid to all demand plans, and (2) are dominant solutions, i.e., the configurations satisfy the condition (1) and achieve the optimal values for $R^{\max }$ and $m$.

Step 4. Selection of related configurations: we determine the affinity degree between each pair of dominant line configurations (resulting from Step 3), and whether this affinity degree is equal or greater than a previously fixed value $\left(A\left(\vec{S}_{n}, \vec{S}_{n^{\prime}}\right) \geq 0.90\right)$, one of these configurations is rejected.

Step 5.Ordination of configurations by the robustness degree: the robustness of each configuration from Step 4 (by index values) is measured and then the configurations are sorted from lowest to highest robustness degree, according to index values $g_{1}, g_{2}, g_{1}{ }^{\max }$ and $g_{2}^{\max }$ 


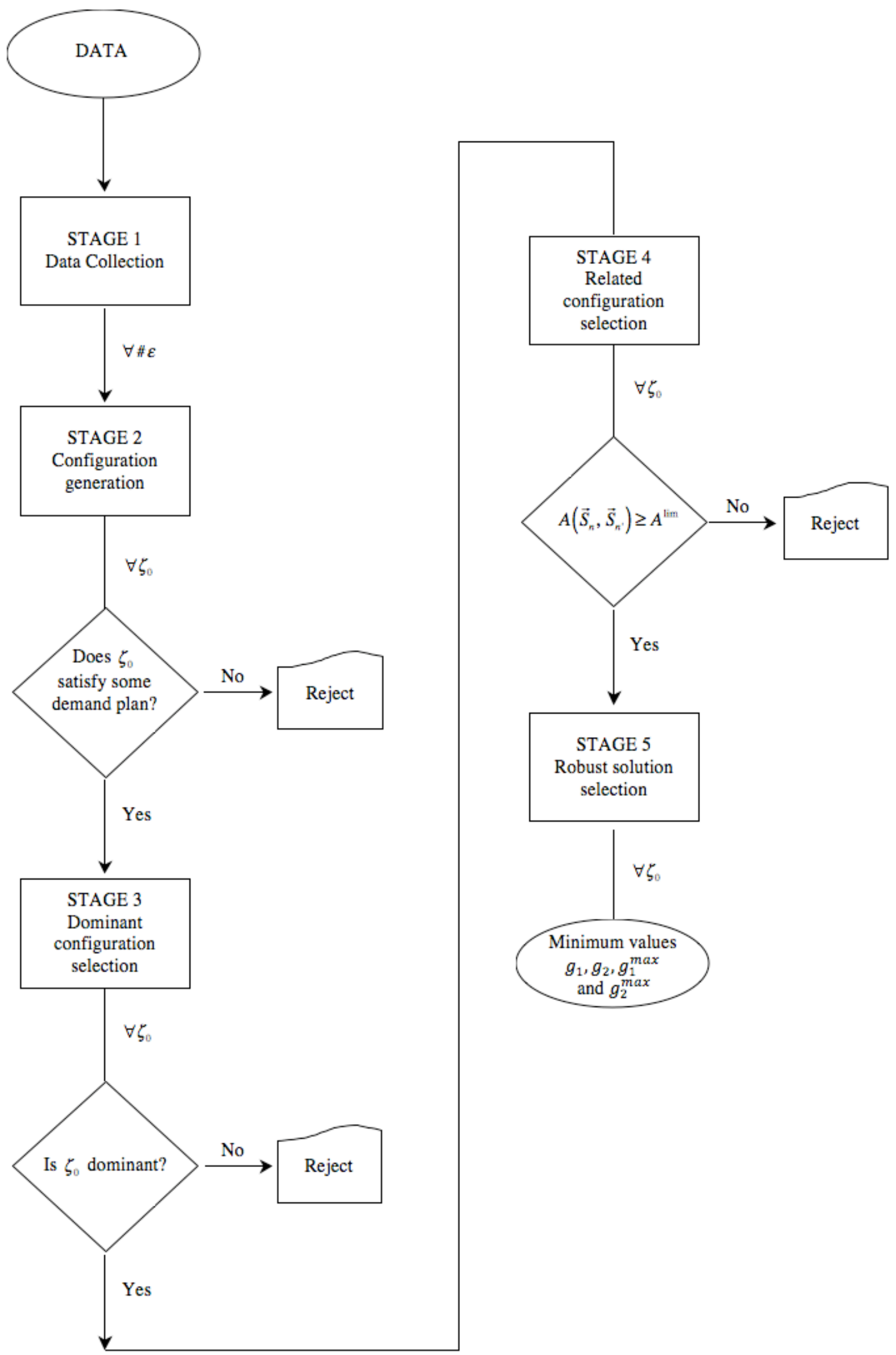

Fig.1.Diagram of decision-making process 


\section{Computational experience}

\subsection{Data set}

To evaluate the impact of considering temporal, spatial and ergonomic risk attributes of workstations on the assembly line balancing problem simultaneously, we have performed an experiment linked to a case study from the power train plant of Nissan Spanish Industrial Operations (NSIO) in Barcelona, Spain.

Specifically, we have used the weighted attribute model with the rectangular function, $\Delta_{R}(T, A, R)$, assigning the same weight to each attribute: workload time, required area and $\operatorname{ergonomic} \operatorname{risk}\left(\mu_{T} T^{\text {med }}=\mu_{A} A^{\text {med }}=\mu_{R} R^{\text {med }}=1 / 3\right)$.

The model has run for different demand plans, different values of maximum allowed ergonomic risk of the workstations and different numbers of workstations on the line. In this way we were able to evaluate the following points:

- The impact of varying the composition of the product mix on the line configuration.

- The similarity degree of the line configurations associated with the different demand scenarios.

- The degree of "robustness" or "resilience" of a configuration facing the variation of the production or demand plans.

The case study is based on a mixed-product assembly line. Specifically, nine types of engines $\left(p_{6}, \ldots, p_{9}\right)$ are assembled in this line with different destinations and assembly features (see Table 3$)$. These types of engines are grouped into three classes: $4 \times 4 \mathrm{~s}\left(p_{1}, \ldots, p_{3}\right)$, vans $\left(p_{4}, p_{5}\right)$ and trucks $\left(p_{6}, \ldots, p_{9}\right)$; but despite their differences, the assembly of the three engine classes requires 378 elementary tasks (including rapid testing). These tasks were grouped into140operations, maintaining the appropriate precedence rules and considering both the maximum available area and the workload times of workstations. Hence, the aggregation of these 140 operations into different workstations of the line, at the time of balancing, was made easier.

Given a global demand, the partial demand for each one of the nine types of engines is not homogeneous in time and is not equal for each one. Thus, although the daily production capacity is kept constant, the line must be able to adapt to different demand plans based on the partial demands of each engine type. As a result, each production program must correspond to a set of average operation times (Chica et. al., 2012) weighted by the demand of the nine 
types of engines. In short, the change in production mix affects the weighted duration of each operation involved in the process and therefore may require a rebalancing of the line.

Because of this, a set $\mathrm{E}$ of nine instances that correspond to different production mixes have been selected to solve the problem studied in this paper (see Table 3). The engine line must satisfy a daily total demand of 270 units. To achieve this daily production, the plant runs on two eight-hour shifts, although effective daily working time per shift is 6 hour and 45 minutes taking into account compulsory breaks and other stoppages. Thus, the resulting cycle time $(c)$ is $180 \mathrm{~s}$.

Table 3: Daily production (units) of engine types for each demand plan $(\# \varepsilon)$.

\begin{tabular}{c|c|ccccccccc} 
& & \multicolumn{10}{|c}{ Demand plan $(\# \varepsilon)$} \\
Family & & $\# 1$ & $\# 2$ & $\# 3$ & $\# 6$ & $\# 9$ & $\# 10$ & $\# 11$ & $\# 12$ & $\# 18$ \\
\hline $4 \times 4$ & $p_{1}$ & 30 & 30 & 10 & 50 & 70 & 10 & 10 & 24 & 60 \\
& $p_{2}$ & 30 & 30 & 10 & 50 & 70 & 10 & 10 & 23 & 60 \\
& $p_{3}$ & 30 & 30 & 10 & 50 & 70 & 10 & 10 & 23 & 60 \\
\hline VAN & $p_{4}$ & 30 & 45 & 60 & 30 & 15 & 105 & 15 & 45 & 30 \\
& $p_{5}$ & 30 & 45 & 60 & 30 & 15 & 105 & 15 & 45 & 30 \\
\hline Trucks & $p_{6}$ & 30 & 23 & 30 & 15 & 8 & 8 & 53 & 28 & 8 \\
& $p_{7}$ & 30 & 23 & 30 & 15 & 8 & 8 & 53 & 28 & 8 \\
& $p_{8}$ & 30 & 22 & 30 & 15 & 7 & 7 & 52 & 27 & 7 \\
& $p_{9}$ & 30 & 22 & 30 & 15 & 7 & 7 & 52 & 27 & 7 \\
\hline
\end{tabular}

Obviously, for each one of the selected instances, the processing times as well as the ergonomic risk of the 140 operations have been calculated based on the production amounts of the different types of engines. Table A.1 (see Appendix) shows these calculated processing times, the required area for each operation $\left(a_{j}\right)$ and the category for each task with respect to the physical risk factor $\left(\chi_{j}\right)$.

Finally, to implement the experiment, a Mathematical Programming Solver (the Solver CPLEX v11.0) was used on a MacPro computer with an Intel Xeon 3.0 GHz CPU and 2 GB RAM using Windows XP with a CPU time limit of $7200 s$.

\subsection{Obtaining line configurations through the balancing model by attributes}

The aim of the first phase of the computational experience is to obtain the best line configurations on the basis of the balance of the attributes (workload time, space and ergonomic risk), and taking into account several scenarios for the demand mix. Specifically, the objective is to improve the ergonomic conditions from an initial line configuration with 21 workstations ( $m=21$ ) (Bautista and Pereira, 2007). This reference 
configuration presents an ergonomic risk ranging from 522 to $531 e-s$ (maximum risk of the line given by the configurations obtained for $\# \varepsilon=11$ and $\# \varepsilon=10$ instances, respectively) and those values are not acceptable industrially if we consider a moderate ergonomic risk as condition what means a risk category of the line lower or equal to 2 .

From nine selected demand plans ( $\# \varepsilon=1,2,3,6,9,10,11,12,18)$, a maximum cycle time of $c=180 \mathrm{~s}$, and a maximum are a available of $A=400 \mathrm{~cm}$, we determine whether it is possible to find a candidate line configuration throught the $M_{-} A A L B{ }_{-} \Delta_{R}(T, A, R)$ model. In effect, we tried to find a solution by limiting the CPU time of $7200 s$ (MacPro) and setting the following parameters:

- Value range for the workstation number: $m=\{21,22,23,24\}$.

- Value range for the maximum ergonomic risk allowed for lines that corresponds to a risk category, $\chi_{\phi}$, comprised between 2 and 2.44 , where $R_{\phi}^{\max }=\chi_{\phi} \cdot c$ :

$$
R_{\phi}^{\max }=\{360,370,380,390,400,410,420,430,440\} \text { ergo-seconds }(e-s) .
$$

Therefore, considering the number of selected instances and the sweep of the number of workstations and maximum ergonomic risk $(9 \times 4 \times 9)$, this experiment involves 324 executions. These executions of the optimization solver are carried out to obtain line configurations when a solution exists for each data set or to conclude that there is no solution. Obviously, this number could be reduced if we consider that a solution, for a specific demand plan and ergonomic risk, will be feasible if we increase the maximum risk.

In Table 4 , we can see the obtained results. For each pair of $m$ and $R_{\phi}^{\max }$ values, we indicate the demand plans $(\# \varepsilon)$ for which the solver has found a line configuration solution. For example, for $m=23$ and $R_{\phi}^{\max }=360 e-s$, the $M_{-} A A L B_{-} \Delta_{R}(T, A, R)$ model has found a feasible solution for each demand plan.

Table 4: Demand plans (\# $\varepsilon$ ) satisfied by each pair of $m$ and $R_{\phi}^{\max }$ values, considering the fixed values $c=180 \mathrm{~s}$ and $A=400 \mathrm{~cm}$.

\begin{tabular}{lccc}
\hline$R_{\phi}^{\max }$ & $m=21$ & $m=22$ & $m=23 ; 24$ \\
\hline 360 & None & $\# 1, \# 9, \# 10, \# 11, \# 12, \# 18$ & All \\
370 & None & $\# 1, \# 2, \# 3, \# 9, \# 10, \# 11, \# 12, \# 18$ & All \\
380 & None & All & All \\
390 & $\# 1$ & All & All \\
400 & $\# 1$ & All & All \\
410 & $\# 1, \# 10$ & All & All \\
420 & $\# 1, \# 10, \# 11, \# 12$ & All & All \\
430 & $\# 1, \# 3, \# 9, \# 10, \# 11, \# 12$ & All & All \\
440 & $\# 1, \# 3, \# 6, \# 9, \# 10, \# 11, \# 12, \# 18$ & All & All \\
\hline
\end{tabular}


From Table 4, we can conclude how the production mix composition affects the line balance under the conditions on the temporal, spatial and ergonomic attributes. To begin, the solver only finds solution in 261 of the 324 executions carried out. Specifically, we observe that whether the line has 21 workstations, even though we allow a maximum ergonomic risk of $440 e-s$, the line cannot perform all of the demand plans. Indeed, for $m=21$

and $R_{\phi}^{\max }=440 e-s($ that corresponds to a line category of $2.4 \hat{4})$ the solver finds feasible configurations for all demand plans except for plan number \#2.

Similarly, there are no solution to any instance when the number of workstations is 21 and the maximum ergonomic risk is equal to $380 e-s$ or less. As it shows, when the number of workstations is 22 , the lowest maximum ergonomic risk that provides solutions for all of the instances is $380 e-s$. Finally, we see the lowest number of workstations that allows all range of ergonomic risk is 23 .

The results shown in Table 4 allow us to reject the line configurations with 21 stations (because not satisfy all plans) and 24 stations (because they are dominated by those of 23 stations). However, we cannot a priori reject any line configuration with $m=22$ or $m=23$ because both cases obtain solutions for some or all instances. Accordingly, we can state both sets of configuration are robust (Chica et al., 2016). Notwithstanding the former, we have analyzed all configurations obtained with $m=22$ and $m=23$ workstations in order to determine which line configuration is more strongly robust regarding a set of conditions imposed to the temporal, spatial and ergonomic attributes. To that end the obtained solutions have been denoted with the 6-tuple ( $\left.m, c, A, R^{\max }, \# \varepsilon, C P U\right)$.

\subsection{Selecting the strongly robust line configuration}

Based on the Nissan's scenario, we establish a set of conditions that any configuration must satisfy with regard to the three attributes considered throughout this paper. These conditions are the following:

- C1. Cycle time equal to $c=180 s$. The time of workload assigned to any station of the line must be lower or equal than $180 s$ for all demand plans from the set $\mathrm{E}$.

- C2. Linear area available equal to $A=400 \mathrm{~cm}$. The linear area required by the tasks assigned to any station of the line must be lower or equal than $400 \mathrm{~cm}$ for all demand plans from the set E. 
- C3. Ergonomic risk category of the line equal to $2\left(R^{\max }=360\right)$. The ergonomic risk associated with the workload of any workstation of the line must be lower or equal than $360 e-s$ for all demand plans, from the set E.

- C4. Any configuration must be obtained under the same conditions, that is through the $M_{-} A A L B \_\Delta_{R}(T, A, R)$ model, using the CPLEX Solver (v11.0) on a MacPro (Intel Xeon CPEU, $3.0 \mathrm{GHz}, 2 \mathrm{~GB}$, Win.XP) and with CPU time limit of $7200 s$ for each demand plan $\# \varepsilon \in \mathrm{E}$.

- C5. One solution (line configuration) is strongly robust if all its attributes satisfy the conditions $\mathrm{C} 1, \mathrm{C} 2, \mathrm{C} 3$ and $\mathrm{C} 4$.

- C6. One solution (line configuration) is acceptable industrially ifit (1) is strongly robust in all its attributes and (2) presents the lowest number of workstations.

Once established the conditions and considering the results given by the $M{ }_{-} A A L B \_\Delta_{R}(T, A, R)$ model (Table 4$)$, we have only analyzed the robustness of (1) the solutions obtained for the \#1, \#9, \#10,\#11,\#12 and \#18 demand plans when the number of workstations is $m=22$ and the maximum ergonomic risk is $R^{\max }=360 e-s$; and (2) the configurations corresponding with all the demand plans when the number of workstations is $m=23$ and the maximum ergonomic risk is $R^{\max }=360 e-s$.

For this analysis we have used the $M_{-} A A L B \_\varnothing$ model, defined in subsection 2.4 of this paper. This model has allowed us to check if the solution obtained for a given demand plan is feasible for the rest of demand plans and then, whenever a specific configuration for a demand plan is feasible for any other demand plan, the initial conditions (C1-C6) have been verified (Bautista et al., 2015b, Bautista et al., 2015c).The results obtained by the feasibility model are the following (Table 5).

Table 5: Demand plans $(\# \varepsilon \in \mathrm{E})$ that satisfy the constraints the cycle time $(c=180 s)$, linear area $(A=400 \mathrm{~cm})$ and ergonomic risk $\left(R^{\max }=360 e-s\right)$ given the set of line configurations $\zeta_{0}=1, \ldots, 15$.

\begin{tabular}{l|ccc}
\hline Configuration & $c=180 s$ & $A=400 \mathrm{~cm}$ & $R^{\max }=360 e-s$ \\
\hline$\zeta_{0}=1: s=(22,180,400,360, \# 1,7200)$ & $\# 1, \# 11$ & $\forall \# \varepsilon \in E$ & $\# 1$ \\
$\zeta_{0}=2: s=(22,180,400,360, \# 9,7200)$ & $\forall \# \varepsilon \in E$ & $\forall \# \varepsilon \in E$ & $\# 1, \# 6, \# 9, \# 18$ \\
$\zeta_{0}=3: s=(22,180,400,360, \# 10,7200)$ & $\# 1, \# 2, \# 3, \# 10, \# 12$ & $\forall \# \varepsilon \in E$ & $\# 10$ \\
$\zeta_{0}=4: s=(22,180,400,360, \# 11,7200)$ & $\# 1, \# 11$ & $\forall \# \varepsilon \in E$ & $\# 1, \# 11$ \\
$\zeta_{0}=5: s=(22,180,400,360, \# 12,7200)$ & $\forall \# \varepsilon \in E$ & $\forall \# \varepsilon \in E$ & $\# 1, \# 2, \# 3, \# 6, \# 10, \# 12, \# 18$ \\
$\zeta_{0}=6: s=(22,180,400,360, \# 18,7200)$ & $\forall \# \varepsilon \in E$ & $\forall \# \varepsilon \in E$ & $\# 1, \# 6, \# 18$ \\
$\zeta_{0}=7: s=(23,180,400,360, \# 1,7200)$ & $\forall \# \varepsilon \in E$ & $\forall \# \varepsilon \in E$ & $\forall \# \varepsilon \in E$ \\
$\zeta_{0}=8: s=(23,180,400,360, \# 2,7200)$ & $\forall \# \varepsilon \in E$ & $\forall \# \varepsilon \in E$ & $\forall \# \varepsilon \in E$ \\
\hline
\end{tabular}




\begin{tabular}{lllcc}
\hline$\zeta_{0}=9: s=(23,180,400,360, \# 3,7200)$ & & $\forall \# \varepsilon \in E$ & $\forall \# \varepsilon \in E$ & $\# 1, \# 2, \# 3, \# 10, \# 11, \# 12$ \\
$\zeta_{0}=10: s=(23,180,400,360, \# 6,7200)$ & & $\forall \# \varepsilon \in E$ & $\forall \# \varepsilon \in E$ & $\forall \# \varepsilon \in E$ \\
$\zeta_{0}=11: s=(23,180,400,360, \# 9,7200)$ & $\forall \# \varepsilon \in E$ & $\forall \# \varepsilon \in E$ & $\forall \# \varepsilon \in E$ \\
$\zeta_{0}=12: s=(23,180,400,360, \# 10,7200)$ & $\forall \# \varepsilon \in E$ & $\forall \# \varepsilon \in E$ & $\# 1, \# 2, \# 3, \# 6, \# 10, \# 12, \# 18$ \\
$\zeta_{0}=13: s=(23,180,400,360, \# 11,7200)$ & $\forall \# \varepsilon \in E$ & $\forall \# \varepsilon \in E$ & $\# 11$ \\
$\zeta_{0}=14: s=(23,180,400,360, \# 12,7200)$ & $\forall \# \varepsilon \in E$ & $\forall \# \varepsilon \in E$ & $\# 1, \# 2, \# 3, \# 6, \# 10, \# 12, \# 18$ \\
$\zeta_{0}=15: s=(23,180,400,360, \# 18,7200)$ & $\forall \# \varepsilon \in E$ & $\forall \# \varepsilon \in E$ & $\# 1, \# 6, \# 9, \# 18$ \\
\hline
\end{tabular}

As evidenced in Table 5, no configuration with 22 workstations satisfy all conditions in the set of demand plans studied. Therefore, we select the configurations $\zeta_{0}=7,8,10,11$ as the most strongly robust because they are the only configurations that fulfil the 6 established criteria. However, despite not meeting the 6 selection criteria established, some configurations with 22 stations only violate the ergonomic risk condition slightly, such as the line configuration $\zeta_{0}=5: s=(22,180,400,360,12,7200)$ (see Table 6).

Table 6: Minimal (Min) and maximum (Max) values for the cycle time $t\left(S_{k}\right)$, linear area $a\left(S_{k}\right)$ and ergonomic risk $R\left(S_{k}\right)$ for the workloads $\left(S_{k}\right)$ given by the line configuration $\zeta_{0}=5: s=(22,180,400,360,12,7200)$ for all studied plans $\# \varepsilon \in E$.

\begin{tabular}{c|cccccc}
\hline \multirow{2}{*}{ \# $\in E$} & \multicolumn{2}{|c}{ Cycle time } & \multicolumn{2}{c}{ Linear area } & \multicolumn{2}{c}{ Ergonomic risk } \\
& $\min \left\{t\left(S_{k}\right)\right\}$ & $\max \left\{t\left(S_{k}\right)\right\}$ & $\min \left\{a\left(S_{k}\right)\right\}$ & $\max \left\{a\left(S_{k}\right)\right\}$ & $\min \left\{R\left(S_{k}\right)\right\}$ & $\max \left\{R\left(S_{k}\right)\right\}$ \\
\hline$\# 1$ & 100.00 & 175.000 & 150 & 400 & 140.000 & 360.000 \\
$\# 2$ & 99.937 & 175.903 & 150 & 400 & 139.950 & 359.380 \\
$\# 3$ & 100.726 & 176.248 & 150 & 400 & 139.622 & 358.956 \\
$\# 6$ & 99.130 & 175.516 & 150 & 400 & 140.311 & 359.878 \\
$\# 9$ & 98.341 & 175.617 & 150 & 400 & 140.639 & 360.302 \\
$\# 10$ & 100.519 & 178.915 & 150 & 400 & 139.506 & 357.169 \\
$\# 11$ & 100.952 & 175.259 & 150 & 400 & 139.706 & 360.669 \\
$\# 12$ & 100.236 & 175.785 & 150 & 400 & 139.839 & 359.409 \\
$\# 18$ & 100.000 & 175.795 & 150 & 400 & 140.450 & 359.780 \\
\hline Min & 98.341 & 175.000 & 150 & 400 & 139.506 & 357.169 \\
Max & 100.952 & 178.915 & 150 & 400 & 140.639 & 360.669 \\
\hline
\end{tabular}

As we can see, the configuration $\zeta_{0}=5: s=(22,180,400,360,12,7200)$ shown in Table 6 , is feasible and fulfills the 6 criteria for all demand plans, except for the \#9 and \#11 demand plans, which present a maximum ergonomic risk slightly greater than the admisible risk. Therefore, if we are strict with the conditions we must reject the configurations with 22 workstations.

In essence, at this point, we have rejected 225 of the 261 solutions obtained with the $M_{-} A A L B \_\Delta_{R}(T, A, R)$ model, selecting only four line configurations with 23 workstations, the configurations $\zeta_{0}=7,8,10,11$ that correspond to the demand plans \#1, \#2, \#6 and \#9, respectively. Therefore, and following our objective of obtaining an unique configuration that 
is strongly robust regarding a set of conditions imposed to the temporal, spatial and ergonomic attributes, next we analyze the four selected configurations through the affinity degree and the indices of robustness defined in section 3 .

\subsection{Similarity degree and "robustness" or "resilience" degree of a configuration regarding the demand variations}

In the previous phase of the experiment, the configurations with $m=23$ obtained for the demand plans \#1, \#2, \#6 and \#9 have been selected as valid configurations for all studied instances. These configurations satisfy all constraints $(m=23, c=180 \mathrm{~s}, \mathrm{~A}=400 \mathrm{~cm}$ and $\left.R_{\phi}^{\max }=360 e-s\right)$, although processing times and ergonomic risks are different. Indeed Table 7 shows these configuration features, specifically shows the assigned operations or workload, $S_{k}$, the processing time, $T$, linear area, $A$, and ergonomic risk, $R$, by workstation.

Table7: Configurations $\zeta_{0}=7,8,10,11$ given by $M_{-} A A L B{ }_{-} \Delta_{R}(T, A, R)$ for the demand plans \#1, \#2, \#6 and \#9 when $m=23, c=180 \mathrm{~s}, A=400 \mathrm{~cm}$ and $R_{\phi}^{\max }=360 e-s$ are considered.

\begin{tabular}{|c|c|c|c|c|c|c|c|c|}
\hline \multirow[b]{2}{*}{$k$} & \multicolumn{4}{|c|}{$\zeta_{0}=7: s=(23,180,400,360, \# 1,7200)$} & \multicolumn{4}{|c|}{$\zeta_{0}=8: s=(23,180,400,360, \# 2,7200)$} \\
\hline & Assigned operations $S_{k}$ & $T$ & $A$ & $R$ & Assigned operations $S_{k}$ & $T$ & $A$ & $R$ \\
\hline 1 & $1,3,10$ & 110.00 & 400 & 140.00 & $1,9,10$ & 109.58 & 400 & 159.60 \\
\hline 2 & $5,8,9,11,13,14,18$ & 130.00 & 400 & 225.00 & $11,13,14,17,19,20$ & 129.52 & 400 & 244.19 \\
\hline 3 & $4,616,19,21$ & 138.00 & 400 & 156.00 & $5,7,15,16,18,21$ & 125.80 & 400 & 231.60 \\
\hline 4 & $15,17,20,25,26,27$ & 133.00 & 400 & 266.00 & $3,4,22,25,26,27$ & 126.91 & 350 & 174.14 \\
\hline 5 & $7,22,23,24,28,29$ & 129.00 & 400 & 258.00 & $6,23,24,28$ & 127.33 & 350 & 194.40 \\
\hline 6 & $30,31,32,33,35,36,37$ & 115.00 & 400 & 280.00 & $2,8,29,30,31$ & 120.01 & 400 & 240.03 \\
\hline 7 & $34,38,39,40,41,42$ & 100.00 & 400 & 300.00 & $32,33,34,35,36,37$ & 99.91 & 350 & 259.71 \\
\hline 8 & $43,44,45,46,48,59$ & 110.00 & 350 & 330.00 & $38,39,40,41,42,59$ & 94.81 & 350 & 284.42 \\
\hline 9 & $12,47,49,51,55,60$ & 120.00 & 200 & 345.00 & $43,44,45,46,47$ & 105.48 & 350 & 316.43 \\
\hline 10 & $50,52,53,54,56,57,58,64$ & 125.00 & 200 & 345.00 & $12,48,49,52,53,55$ & 119.86 & 150 & 344.53 \\
\hline 11 & $61,62,63,66,67$ & 130.00 & 300 & 260.00 & $50,51,54,56,57,58,60,64$ & 125.26 & 250 & 345.55 \\
\hline 12 & $65,68,69,70,71,72$ & 125.00 & 400 & 250.00 & $61,62,63,66,67$ & 130.23 & 300 & 260.45 \\
\hline 13 & $73,74,75,77,78,79$ & 120.00 & 400 & 240.00 & $65,68,71,72,73$ & 125.03 & 400 & 250.06 \\
\hline 14 & $76,80,81,82,83,84,88,90$ & 115.00 & 375 & 275.00 & $69,70,74,75,77,78,79$ & 119.76 & 400 & 239.51 \\
\hline 15 & $2,85,89$ & 115.00 & 300 & 270.00 & $76,80,81,82,83,84,88,90$ & 114.81 & 375 & 274.45 \\
\hline 16 & $86,87,91,92,93,99$ & 130.00 & 250 & 345.00 & $85,86,87,89,91,92$ & 130.26 & 300 & 345.65 \\
\hline 17 & $98,100,101,102,103$ & 150.00 & 200 & 300.00 & $98,99,100,101,103$ & 159.20 & 150 & 348.00 \\
\hline 18 & $\begin{array}{l}104,108,109,111,112,113 \\
114,115,116\end{array}$ & 135.00 & 200 & 275.00 & $\begin{array}{l}102,104,108,109,111,112,113 \\
114,115,116\end{array}$ & 154.34 & 250 & 313.70 \\
\hline 19 & $106,117,118,131,132,134$ & 155.00 & 300 & 265.00 & $106,117,118,131,132,134$ & 155.83 & 300 & 266.48 \\
\hline 20 & $105,107,119,120,121,122$ & 140.00 & 300 & 270.00 & $105,107,119,120,121,122,123$ & 149.75 & 350 & 279.52 \\
\hline 21 & $\begin{array}{l}110,123,124,125,126,128 \\
129,135\end{array}$ & 145.00 & 350 & 235.00 & $124,125,126,127,128,129,135$ & 139.91 & 350 & 234.69 \\
\hline 22 & $127,130,133,136,137,138$ & 145.00 & 325 & 245.00 & $93,110,130,133,136,137,138$ & 150.06 & 325 & 264.95 \\
\hline 23 & $94,95,96,97,139,140$ & 175.00 & 300 & 270.00 & $94,95,96,97,139,140$ & 175.90 & 300 & 270.95 \\
\hline
\end{tabular}

\begin{tabular}{|c|c|c|c|c|c|c|c|c|}
\hline \multirow[b]{2}{*}{$k$} & \multicolumn{4}{|c|}{$\zeta_{0}=10: s=(23,180,400,360, \# 6,7200)$} & \multicolumn{4}{|c|}{$\zeta_{0}=11: s=(23,180,400,360, \# 9,7200)$} \\
\hline & Assigned operations $S_{k}$ & $T$ & $A$ & $R$ & Assigned operations $S_{k}$ & $T$ & $A$ & $R$ \\
\hline 1 & $1,9,10$ & 110.24 & 400 & 160.10 & $1,3,10$ & 110.38 & 400 & 140.44 \\
\hline 2 & $3,5,7,8,11,13$ & 124.26 & 400 & 194.30 & $5,7,9,11,13,14$ & 124.40 & 400 & 214.16 \\
\hline 3 & $14,15,17,19,20,21$ & 112.74 & 400 & 225.47 & $15,16,17,19,20,21$ & 111.10 & 400 & 222.20 \\
\hline 4 & $16,18,22,24,25,26,27$ & 125.04 & 350 & 250.08 & $18,22,23,24,25,26,27$ & 123.78 & 350 & 247.55 \\
\hline 5 & $4,23,28,29,30$ & 122.51 & 400 & 184.21 & $4,8,28,29,30$ & 126.02 & 400 & 190.34 \\
\hline 6 & $6,31,32,33,34$ & 126.31 & 350 & 231.78 & $6,31,32,33,34$ & 127.55 & 350 & 233.76 \\
\hline 7 & $2,35,36,37$ & 119.72 & 350 & 259.62 & $2,35,36,37$ & 119.27 & 350 & 258.87 \\
\hline 8 & $38,39,40,41,42,59$ & 93.96 & 350 & 281.88 & $38,39,40,41,42,59$ & 93.13 & 350 & 279.39 \\
\hline
\end{tabular}




\begin{tabular}{|c|c|c|c|c|c|c|c|c|}
\hline 9 & $43,44,45,46,47$ & 105.67 & 350 & 317.01 & $43,44,45,46,47$ & 105.87 & 350 & 317.62 \\
\hline 10 & $48,49,50,51,54,60$ & 115.21 & 200 & 345.64 & $12,48,49,50,55,56,60$ & 120.02 & 250 & 345.10 \\
\hline 11 & $12,52,53,55,56,57,58,64$ & 129.76 & 200 & 344.46 & $51,52,53,54,57,58,64$ & 124.81 & 150 & 345.02 \\
\hline 12 & $61,62,63,66,67$ & 128.58 & 300 & 257.16 & $61,62,63,66,67$ & 126.96 & 300 & 253.92 \\
\hline 13 & $65,68,71,72,73$ & 124.84 & 400 & 249.68 & $65,68,69,70,71,72$ & 124.35 & 400 & 248.70 \\
\hline 14 & $69,70,74,75,77,78,79$ & 120.83 & 400 & 241.66 & $73,74,75,77,78,79$ & 122.18 & 400 & 244.36 \\
\hline 15 & $76,80,81,82,83,84,88,90$ & 113.83 & 375 & 272.41 & $76,80,81,82,83,84,88,90$ & 112.85 & 375 & 270.37 \\
\hline 16 & $85,86,87,89,91,92$ & 130.18 & 300 & 345.49 & $85,86,87,89,91,92$ & 130.07 & 300 & 345.22 \\
\hline 17 & $98,99,100,101,102$ & 150.22 & 150 & 330.34 & $98,99,100,101,102$ & 150.95 & 150 & 332.10 \\
\hline 18 & $\begin{array}{l}103,104,108,109,111,112, \\
113,114,115,116\end{array}$ & 165.54 & 250 & 336.10 & $\begin{array}{l}103,104,108,109,111,112,113 \\
114,115,116\end{array}$ & 166.99 & 250 & 338.99 \\
\hline 19 & $106,117,118,131,132,134$ & 155.51 & 300 & 265.30 & $106,107,117,118,131,132,134$ & 160.12 & 300 & 278.99 \\
\hline 20 & $\begin{array}{l}105,107,119,120,121,122 \\
123\end{array}$ & 150.06 & 350 & 280.16 & $105,119,120,121,122,123$ & 145.39 & 350 & 265.88 \\
\hline 21 & $\begin{array}{l}124,125,126,135 \\
136,137,138\end{array}$ & 154.65 & 350 & 244.32 & $\begin{array}{l}110,124,125,126,128,135, \\
136,137\end{array}$ & 154.33 & 350 & 243.80 \\
\hline 22 & $\begin{array}{l}93,110,127,128,129,130, \\
133\end{array}$ & 134.85 & 325 & 255.09 & $93,127,129,130,133,138$ & 134.74 & 325 & 255.41 \\
\hline 23 & $94,95,96,97,139,140$ & 175.52 & 300 & 271.09 & $94,95,96,97,139,140$ & 175.17 & 300 & 271.25 \\
\hline
\end{tabular}

However, we can improve the decision-making process through both qualitative and quantitative selection criteria, such as the similarity degree between workstations defined in section 3.1 (equation 44). This index gives us an idea of the changes of line configuration in case of a change in the demand plan. Indeed, the higher the index value, the lower the changes at workstations of the line, such as changes of tools, movements of shelf and equipment, or training of workers to adapt them to the new situation.

Table 8 shows the similarity degree between two configurations. It should be noted that the configuration most related with the rest is the one with the greatest value in the average affinity index $\overline{\mathrm{A}}$, and obviously the index is 1 when a configuration is compared with itself. Therefore, we can stand out the configurations $\zeta_{0}=10$ and $\zeta_{0}=11$ (corresponding to the demand plans \#6 and \#9) as the most similar solutions and also the closest to configurations $\zeta_{0}=7$ and $\zeta_{0}=8$ (instances \#1 and \#2 respectively).

Table 8: Affinity index, $A\left(\vec{S}_{n}, \vec{S}_{n^{\prime}}\right)$, between the four configurations selected in the previous phase.

\begin{tabular}{cccccc}
\hline$A\left(\vec{S}_{n}, \vec{S}_{n^{\prime}}\right)$ & $\zeta_{0}=7$ & $\zeta_{0}=8$ & $\zeta_{0}=10$ & $\zeta_{0}=11$ & $\overline{\mathrm{A}}$ \\
\hline$\zeta_{0}=7: s=(23,180,400,360, \# 1,7200)$ & 1.000 & 0.470 & 0.448 & 0.471 & 0.597 \\
$\zeta_{0}=8: s=(23,180,400,360, \# 2,7200)$ & 0.470 & 1.000 & 0.748 & 0.735 & 0.738 \\
$\zeta_{0}=10: s=(23,180,400,360, \# 6,7200)$ & 0.448 & 0.748 & 1.000 & 0.870 & 0.767 \\
$\zeta_{0}=11: s=(23,180,400,360, \# 9,7200)$ & 0.471 & 0.735 & 0.870 & 1.000 & 0.769 \\
\hline
\end{tabular}

On the other hand, we get the indices $g_{1}, g_{2}, g_{1}^{\max }$ and $g_{2}^{\max }$ (Table 9), which are defined in Section 3. Thanks to them we can measure the robustness of each selected configuration and therefore we can choose the stronger configuration against possible changes in demand. 
Table 9. Index values, $g_{1}, g_{2}, g_{1}^{\max }$ and $g_{2}^{\max }$, for attributes $T, A$ and $R$, from the configurations $\zeta_{0}=7,8,10,11$.

\begin{tabular}{|c|c|c|c|c|c|c|c|c|c|c|c|c|}
\hline \multirow{2}{*}{$\zeta_{0}$} & \multicolumn{3}{|c|}{$g_{1}$} & \multicolumn{3}{|c|}{$g_{2}$} & \multicolumn{3}{|c|}{$g_{1}^{\max }$} & \multicolumn{3}{|c|}{$g_{2}^{\max }$} \\
\hline & 0,1 & 0 , & $, R)$ & 0 , & 0, & $\left(\zeta_{0}, R\right)$ & $\left(\zeta_{0}, T\right)$ & $\left(\zeta_{0}, A\right.$ & $\left(\zeta_{0}, R\right)$ & $\left(\zeta_{0}, T\right)$ & $\left(\zeta_{0}, A\right)$ & $\left(\zeta_{0}, R\right)$ \\
\hline 7 & 0.353 & 0.219 & 0.298 & 0.050 & 0.097 & 0.065 & 0.255 & 0.179 & 0.221 & 0.036 & 0.080 & 0.048 \\
\hline 8 & 0.353 & 0.219 & 0.321 & 0.059 & 0.083 & 0.074 & 0.255 & 0.179 & 0.221 & 0.043 & 0.068 & 0.055 \\
\hline 10 & 0.353 & 0.219 & 0.29 & 0.059 & 0.083 & 0. & 55 & 0.179 & 0.221 & 0.043 & 0.068 & 0.055 \\
\hline 11 & 0.353 & 0.219 & 296 & .059 & 0.083 & 0.074 & 0.255 & 0.179 & 0.221 & 0.043 & 0.068 & 0.055 \\
\hline
\end{tabular}

From Table9, within the $g_{1}$ index (measure of maximum excess with respect to the average value of the attribute), the configurations with lower maximum ergonomic risk excess are $\zeta_{0}=11,10,7$. However, if we consider the $g_{2}$ index, the configuration with the lowest overall excess is the number $\zeta_{0}=7$, which corresponds to the demand plan \#1 that presents a completely balanced production mix.

\subsection{Summary of computational experience}

To summarize, after the data collection of demand plans linked with the Nissan's engine plant (Stage 1), we have carried out the making-decision process (defined in section 3) to select strong configurations in front of production mix variations and ergonomic risk level.

Subsequently, on stage 2, a line configuration for each daily engine demand, given a range of maximum ergonomics risk factors $\left(R_{\phi}^{\max }\right)$, has been obtained. Then, we have executed the mathematical model in order to find the solutions for all demand plans, considering attribute values. After running 324 times the $M_{-} A A L B_{-} \Delta_{R}(T, A, R)$ model, 15 line configurations have been selected. The selected configurations correspond to configurations with $m=22$ and $m=23$ workstations and an ergonomic risk of $R_{\phi}^{\max }=360 e_{-} s$.

At stage 3, we have chosen the configurations, among the selected in the previous step, that satisfy all studied demand plans and a set of six conditions pre-set. As a result, we have obtained the 4 configurations that satisfy all requirements set by the company. These line configurations are shown in Table 7.

At stage 4, we have determined the affinity index, defined at equation (44), for the purpose of measuring the similarity between two line configurations. Thanks to this criterion, we have been able to select the most similar configuration to all candidates. 
Finally, at stage 5, as alternative to previous stage, we have determined the criterion for discriminating those configurations that generate greater maximum excesses of temporal, spatial and ergonomic attributes with respect to the average values of these. This alternative has been supported by the indices $g_{1}, g_{2}, g_{1}^{\max }$ and $g_{2}^{\max }$, which are calculated according equations (51) - (56).

Briefly, Table 10 shows the selected configurations according the proposed decision-making process, which is based on the different criteria defined in this paper.

Table 10: Selected configurations.

\begin{tabular}{|c|c|c|}
\hline Stages & Criteria & Selected Configurations \\
\hline \multirow{7}{*}{1} & \multirow{7}{*}{$\zeta_{0}$ feasible with $\min \left\{m, R_{\phi}^{\max }\right\}$} & $\zeta_{0}=1: s=(22,180,400,360, \# 1,7200)$ \\
\hline & & $\zeta_{0}=2: s=(22,180,400,360, \# 9,7200)$ \\
\hline & & $\zeta_{0}=3: s=(22,180,400,360, \# 10,7200)$ \\
\hline & & $\zeta_{0}=4: s=(22,180,400,360, \# 11,7200)$ \\
\hline & & $\zeta_{0}=5: s=(22,180,400,360, \# 12,7200)$ \\
\hline & & $\zeta_{0}=6: s=(22,180,400,360, \# 18,7200)$ \\
\hline & & $\zeta_{0}=7, \ldots, 15: s=(23,180,400,360, \# \varepsilon \in \mathrm{E}, 7200)$ \\
\hline \multirow{4}{*}{2} & \multirow{2}{*}{$\zeta_{0}$ satisfy C1-C6 conditions $\forall \varepsilon \in \mathrm{E}$} & $\zeta_{0}=7: s=(23,180,400,360, \# 1,7200)$ \\
\hline & & $\zeta_{0}=8: s=(23,180,400,360, \# 2,7200)$ \\
\hline & \multirow[t]{2}{*}{$\left(c=180 \mathrm{~s} ; A=400 \mathrm{~cm} ; R^{\max }=360 e-s ; C P U_{\text {Limit }}=7200 \mathrm{~s}\right)$} & $\zeta_{0}=10: s=(23,180,400,360, \# 6,7200)$ \\
\hline & & $\zeta_{0}=11: s=(23,180,400,360, \# 9,7200)$ \\
\hline \multirow[t]{2}{*}{3} & \multirow[t]{2}{*}{ Average affinity: $\max A\left(\zeta_{0}\right)$} & $\zeta_{0}=10: s=(23,180,400,360, \# 6,7200)$ \\
\hline & & $\zeta_{0}=11: s=(23,180,400,360, \# 9,7200)$ \\
\hline \multirow{4}{*}{4} & \multirow{3}{*}{ Maximum excess: $\min g_{1}(T, A, R) ; \min g_{1}^{\max }(T, A, R)$} & $\zeta_{0}=7: s=(23,180,400,360, \# 1,7200)$ \\
\hline & & $\zeta_{0}=10: s=(23,180,400,360, \# 6,7200)$ \\
\hline & & $\xi_{0}=11: s=(23,180,400,360, \# 9,7200)$ \\
\hline & Overall excess: $\min g_{2}(T, A, R) ; \min g_{2}^{\max }(T, A, R)$ & $\xi_{0}=7: s=(23,180,400,360, \# 1,7200)$ \\
\hline
\end{tabular}

\section{Conclusions}

In this paper, we have emphasized the importance of considering the ergonomic risk concept in assembly line balancing problems. We have defined the ergonomic risk concept depending on the ergonomic risk category on the basis of the type of task and its processing time.

Additionally, we have proposed a classification of risk category that unifies several assessment methods of risk factors such as postural loads, repetitive movements and manual handling. We have also presented a family of line balancing models that consider temporal and spatial attributes (TSALBP) while incorporating ergonomic risk attributes. Indeed, from a basic feasibility model, we have presented mono-, bi- and tri-objective optimization models 
using elemental functions for the attributes and even 4 models based on weighted attribute functions.

At the same time, we have proposed a methodology whose objective is to obtain a robust line balancing which is capable of meeting the maximum number of scenarios given the demand variation.

For this purpose, we have used a weighted balancing model. Specifically, we have used the $M_{\_} A A L B \_\Delta_{R}(T, A, R)$ model whose objective function focus on weighting temporal, spatial and ergonomic attributes. This linear programming model has been running using the Solver CPLEX v11.0.

Through the Solver CPLEX v11.0, we have performed a computational experiment based on a case study from the Nissan's engine plant in Barcelona. In this way, we have focused on activities related to the automotive industry, where the tasks require effort and attention by the workers. Obviously, this study may also be useful in heavy industries of metallurgy sector but we think this is not necessary in other industries where such effort and attention are not required, and therefore the effect of incorporating ergonomic risk may be insignificant. We have defined four different criteria to determine which configurations are more robust against changes in demand plans. These criteria measure the feasibility degree of a configuration, given a demand plan, and they are: (1) the feasibility of configurations given a set of conditions regarding the values of attributes; (2) the feasibility of a configuration against all demand plans; (3) the similarity degree between alternative configurations; and (4) the maximum and average excesses of the attributes (temporal, spatial and ergonomic) with respect to their average values.

The decision-making process has allowed us to select the configurations corresponding to the demand plans \#1, \#6 and \#9 with 23 workstations as the most robust configurations because they satisfy the requirements of the company and present the best values for the affinity and robustness indices. Therefore, the configurations \#1, \#6 and \#9are the solutions that can be adapted to any demand plan without falling into excessive change costs at the line. To sum up, if ergonomic risk increases, workers may suffer injuries that can lead to chronic diseases. These diseases suppose great costs not only to the company but also to the society. On the other hand, if the number of workstations increases in order to improve the health of workers, the configuration line will change and it will also mean a cost. Therefore, it is important to reach an appropriate balance between the disease risk and the changes in the line. 
For future research, we propose: (1) to asses the multi-objective models with elemental functions (cycle, area and risk) using MILP and metaheuristic procedures and (2) to create new models that integrate the concept of robustness of the line configuration against the demand plan variation.

\section{Acknowledgements}

This work was funded by the Ministerio de Economía y Competitividad (Spanish Government) through the PROTHIUS-III (DPI2010-16759) and FHI-SELM2 (TIN2014-57497-P) projects.

\section{References}

Bautista, J.; Batalla, C.; Alfaro, R.; Cano-Pérez, A., 2013. Extended Models for TSALBP with Ergonomic Risk Constraints, Comunicación, IFAC Conference on Manufacturing Modelling, Management and Control (MIM 2013); Saint Petersburg (Rusia).

Bautista, J.; Batalla, C.; Alfaro, R., 2012. Incorporating Ergonomics Factors into the TSALBP, Comunicación, Proceedings(CD), International Conference on Advances in Production Management Systems (APMS 2012), Rodas (Grecia)

Bautista, J.; Batalla-García, C.; Alfaro-Pozo, R., 2015a. Eficiencia en líneas de montaje de modelos mixtos con múltiples atributos en función del mix de producción. In:

DOI:10.13140/RG.2.1.1052.6887 Report number: OPE-WP.2015/07, Universitat Politècnica de Catalunya-Barcelona-Tech.

Bautista, J.; Batalla-García, C.; Alfaro-Pozo, R., 2015b. Análisis de la robustez de configuraciones de líneas de montaje con múltiples atributos. Parte I. In:

DOI:10.13140/RG.2.1.2243.2725 Report number: OPE-WP.2015/09, Universitat Politècnica de Catalunya-Barcelona-Tech.

Bautista, J., Pereira J., 2007. Ant algorithms for a time and space constrained assembly line balancing problem.European Journal of Operational Research, vol. 177, 2016-2032.

Baybars, I., 1986. A survey of exact algorithms for the simple assembly line balancing problem. Management Science, 32, 909-932.

Battaïa, O., Dolgui, A. 2012. Reduction approaches for a generalized line balancing problem. Computers \& Operations Research, 39, 2337-2345.

Battaïa, O., Dolgui, A. 2013. A taxonomy of line balancing problems and their solution approaches. International Lournal ofProduction Economics, 142, 259-277.

Becker, C., Scholl, A., 2006. A survey on problems and methods in generalized assembly line balancing. European Journal of Operational Research, 168, 694-715. 
Chica, M., Bautista, J.,Cordón, O., Damas, S., 2016. A multiobjective model and evolutionary algorithms for robust time and space assembly line balancing under uncertain demand. Omega 58, 55-68.

Chica, M., Cordón, O., Damas, S., Bautista, J., 2010. Multiobjective constructive heuristics for the $1 / 3$ variant of the time and space assembly line balancing problem: ACO and random greedy search. Information Sciences, 180, 3465-3487.

Chica, M., Cordón, O., Damas, S., Bautista, J., 2011. Including different kinds of preferences in a multiobjective and algorithm for time and space assembly line balancing on different Nissan scenarios. Expert Systems with Applications, 38, 709-720.

Chica, M.; Cordón, O.; Damas, S.; Bautista, J., 2012. Multiobjective memetic algorithms for time and space assembly line balancing, Engineering Applications of Artificial Intelligence, $25,254-273$.

Colombini, D., Occhipinti, E., Grieco, E., 2002. Risk Assessment and Management of Repetitive Movements and Exertions of Upper Limbs Job Analysis, Ocra Risk Indices, Ocra Risk Indices, Prevention Strategies and Design Principles. Elsevier Ergonomics Book Series, vol. 2, ISBN: 978-0-08-044080-4.

Hignett, S., McAtamney, L., 2000. Rapid Entire Body Assessment (REBA). Applied Ergonomics, 31, 201-205.

Karhu O., Kansi P., Kuorinka L., 1977. Correcting working postures in industry: A practical method for analysis. Applied Ergonomics, 8, 199-201.

McAtamney, L., Corlett, E. N., 1993. RULA: a survey method for the investigation of workrelated upper limb disorders. Applied Ergonomics, 24, 91-99.

Otto, A., Scholl, A., 2011. Incorporating ergonomic risks into assembly line balancing. European Journal of Operational Research, 212, 277-286.

Salveson, M.E., 1955. The assembly line balancing problem. Journal of Industrial Engineering, 6, 18-25.

Scholl, A., Fliedner, M., Boysen, N., 2010. Absalom: Balancing assembly lines with assignment restrictions. European Journal of Operational Research, 200, 688-701.

Snook, S.H., Ciriello, V.C., 1991. The design of manual handling tasks: revised tables of maximum acceptable weights and force. Ergonomics, 34, 1197-1213.

Waters, T. R., Baron, S. L., Kemmlert, K., 1997. Accuracy of measurements for the revised NIOSH lifting equation. Applied Ergonomics, 29, 433-438. 


\section{APPENDIX A}

Table A.1: Data corresponding to the nine selected demand plans. Operations necessary to assemble any engine $(|J|=140)$ with their precedence relationships $\left(P_{j}, \forall j \in J\right)$, operation processing times of each demand plan $\left(t_{j}, \forall j \in J ; \forall \# \varepsilon \in \mathrm{E}\right)$ in seconds $(s)$, area required by each operation $\left(a_{j}, \forall j \in J\right)$ in centimeter $(\mathrm{cm})$ and ergonomic risk category associated with each operation $\left(\chi_{j}, \forall j \in J\right)$.

\begin{tabular}{|c|c|c|c|c|c|c|c|c|c|c|c|c|}
\hline \multirow[t]{2}{*}{$j$} & \multirow{2}{*}{$P_{j}$} & \multicolumn{9}{|c|}{$t_{j}(\# \varepsilon)$} & \multirow[t]{2}{*}{$a_{j}$} & \multirow[t]{2}{*}{$\chi_{j}$} \\
\hline & & 1 & 2 & 3 & 6 & 9 & 10 & 11 & 12 & 18 & & \\
\hline 1 & -- & 60.00 & $\begin{array}{l}59.56 \\
\end{array}$ & $\begin{array}{l}58.76 \\
\end{array}$ & 60.38 & 61.18 & 57.45 & 60.05 & $\begin{array}{l}59.44 \\
\end{array}$ & 60.56 & 300 & 1 \\
\hline 2 & 3,31 & 75.00 & 75.03 & 75.44 & 74.6 & 74.18 & 75.51 & 75.39 & 75.18 & 74.4 & 200 & 2 \\
\hline 3 & 1 & 20.00 & 19.78 & 20.11 & 19.46 & 19.13 & 19.46 & 20.76 & 19.96 & 19.18 & 50 & 1 \\
\hline 4 & 3,5 & 60.00 & 59.9 & 59.02 & 60.81 & 61.69 & 58.75 & 59.25 & 59.63 & 61.2 & 100 & 1 \\
\hline 5 & 1 & 20.00 & 19.99 & 20.33 & 19.67 & 19.32 & 20.32 & 20.32 & 20.10 & 19.49 & 50 & 1 \\
\hline 6 & 4,5 & 60.00 & 60.26 & 59.33 & 61.17 & 62.10 & 60.10 & 58.6 & 59.88 & 61.76 & 150 & 1 \\
\hline 7 & 1 & 45.00 & 44.95 & 44.8 & 45.13 & 45.28 & 44.68 & 44.9 & 44.91 & 45.18 & 100 & 2 \\
\hline 8 & 1 & 10.00 & 10.01 & 10.09 & 9.93 & 9.85 & 10.10 & 10.07 & 10.03 & 9.89 & 50 & 2 \\
\hline 9 & 1 & 20.00 & 19.82 & 19.93 & 19.73 & 19.63 & 19.40 & 20.44 & 19.91 & 19.59 & 50 & 2 \\
\hline 10 & 1 & 30.00 & 30.2 & 30.27 & 30.13 & 30.07 & 30.87 & 29.67 & 30.15 & 30.20 & 50 & 2 \\
\hline 11 & 1 & 15.00 & 14.9 & 14.79 & 14.99 & 15.1 & 14.47 & 15.12 & 14.90 & 15,00 & 50 & 2 \\
\hline 12 & 11 & 15.00 & 15.05 & 15.11 & 15,00 & 14.95 & 15.27 & 14.94 & 15.05 & 15,00 & 50 & 2 \\
\hline 13 & 1 & 15.00 & 14.86 & 14.63 & 15.09 & 15.32 & 14.20 & 15.05 & 14.83 & 15.13 & 100 & 1 \\
\hline 14 & 1,13 & 10.00 & 9.93 & 10.02 & 9.84 & 9.75 & 9.81 & 10.22 & 9.98 & 9.76 & 50 & 2 \\
\hline 15 & $9,10,11,13,14$ & 8.00 & 8.1 & 8.06 & 8.14 & 8.17 & 8.35 & 7.77 & 8.05 & 8.20 & 100 & 2 \\
\hline 16 & $9,10,11,13,14$ & 8.00 & 8.03 & 7.91 & 8.16 & 8.28 & 8.01 & 7.81 & 7.98 & 8.23 & 50 & 2 \\
\hline 17 & $9,10,11,13,14$ & 80.00 & 79.8 & 80,00 & 79.6 & 79.4 & 79.40 & 80.6 & 79.91 & 79.4 & 100 & 2 \\
\hline 18 & $9,10,11,13,14$ & 40.00 & 39.7 & 39.5 & 39.88 & 40.09 & 38.58 & 40.44 & 39.76 & 39.84 & 50 & 2 \\
\hline 19 & $9,10,11,13,14$ & 5.00 & 5.01 & 4.97 & 5.05 & 5.09 & 5.00 & 4.93 & 4.99 & 5.08 & 50 & 2 \\
\hline 20 & $9,10,11,13,14$ & 5.00 & 5.03 & 5.05 & 5.01 & 4.99 & 5.14 & 4.96 & 5.03 & 5.01 & 50 & 2 \\
\hline 21 & $9,10,11,13,14$ & 5.00 & 5.02 & 4.94 & 5.1 & 5.17 & 5.01 & 4.88 & 4.99 & 5.15 & 50 & 2 \\
\hline 22 & 26,27 & 7.00 & 6.97 & 6.90 & 7.04 & 7.12 & 6.81 & 6.99 & 6.96 & 7.06 & 50 & 2 \\
\hline 23 & 26,27 & 7.00 & 7.00 & 6.99 & 7.01 & 7.02 & 7.00 & 6.99 & 7.00 & 7.01 & 50 & 2 \\
\hline 24 & 26,27 & 30.00 & 30.14 & 30.27 & 29.98 & 29.86 & 30.66 & 29.91 & 30.14 & 29.99 & 50 & 2 \\
\hline 25 & 26,27 & 30.00 & 30.29 & 30.72 & 29.86 & 29.43 & 31.6 & 29.85 & 30.34 & 29.79 & 50 & 2 \\
\hline 26 & $15,16,17,18,19,20,21$ & 5.00 & 4.96 & 4.88 & 5.04 & 5.12 & 4.77 & 5.00 & 4.95 & 5.06 & 50 & 2 \\
\hline 27 & $15,16,17,18,19,20,21$ & 5.00 & 5,00 & 4.94 & 5.08 & 5.14 & 4.95 & 4.92 & 4.98 & 5.11 & 50 & 2 \\
\hline 28 & $22,23,24,25$ & 30.00 & 29.92 & 30.00 & 29.85 & 29.77 & 29.77 & 30.22 & 29.98 & 29.77 & 100 & 2 \\
\hline 29 & 28 & 10.00 & 9.98 & 10.06 & 9.90 & 9.82 & 9.99 & 10.13 & 10.01 & 9.85 & 50 & 2 \\
\hline 30 & 29 & 15.00 & 14.98 & 15.03 & 14.94 & 14.89 & 14.99 & 15.07 & 15.00 & 14.91 & 100 & 2 \\
\hline 31 & $6,7,8,30$ & 10.00 & 10.02 & 10.2 & 9.85 & 9.67 & 10.27 & 10.12 & 10.07 & 9.77 & 0 & 2 \\
\hline 32 & 31 & 15.00 & 14.9 & 14.84 & 14.97 & 15.03 & 14.56 & 15.11 & 14.91 & 14.95 & 50 & 2 \\
\hline 33 & 32 & 30.00 & 29.77 & 29.3 & 30.25 & 30.72 & 28.62 & 29.97 & 29.68 & 30.37 & 100 & 3 \\
\hline 34 & 32 & 10.00 & 10.11 & 10.14 & 10.07 & 10.04 & 10.47 & 9.82 & 10.08 & 10.11 & 50 & 3 \\
\hline 35 & 36 & 5.00 & 4.99 & 4.98 & 4.99 & 4.99 & 4.94 & 5.02 & 4.99 & 4.99 & 50 & 3 \\
\hline 36 & 32 & 25.00 & 25.1 & 25.29 & 24.94 & 24.75 & 25.61 & 24.95 & 25.14 & 24.9 & 100 & 2 \\
\hline 37 & 32,35 & 15.00 & 15.04 & 14.89 & 15.19 & 15.34 & 15.01 & 14.76 & 14.97 & 15.29 & 0 & 3 \\
\hline 38 & $33,34,36,37$ & 5.00 & 5.01 & 4.92 & 5.11 & 5.20 & 4.95 & 4.88 & 4.98 & 5.16 & 50 & 3 \\
\hline 39 & $33,34,36,37$ & 5.00 & 5.00 & 5.01 & 4.99 & 4.98 & 5.00 & 5.02 & 5.00 & 4.98 & 50 & 3 \\
\hline 40 & $33,34,36,37$ & 5.00 & 4.99 & 5.03 & 4.95 & 4.91 & 5.00 & 5.06 & 5.01 & 4.92 & 50 & 3 \\
\hline 41 & $38,39,40$ & 60.00 & 59.76 & 60.67 & 58.83 & 57.93 & 59.93 & 61.43 & 60.16 & 58.26 & 50 & 3 \\
\hline 42 & $38,39,40$ & 15.00 & 15.07 & 14.96 & 15.18 & 15.29 & 15.17 & 14.74 & 15.01 & 15.27 & 150 & 3 \\
\hline 43 & $38,39,40$ & 15.00 & 14.99 & 14.88 & 15.09 & 15.19 & 14.84 & 14.92 & 14.95 & 15.14 & 150 & 3 \\
\hline 44 & $41,42,43$ & 25.00 & 24.87 & 24.54 & 25.19 & 25.52 & 24.13 & 24.96 & 24.81 & 25.29 & 50 & 3 \\
\hline 45 & $41,42,43$ & 25.00 & 25.20 & 25.28 & 25.14 & 25.06 & 25.90 & 24.65 & 25.15 & 25.20 & 50 & 3 \\
\hline 46 & 44,45 & 5.00 & 5.04 & 5.07 & 5.00 & 4.97 & 5.18 & 4.96 & 5.04 & 5.00 & 50 & 3 \\
\hline 47 & 46 & 35.00 & 35.38 & 35.51 & 35.25 & 35.13 & 36.65 & 34.37 & 35.29 & 35.38 & 50 & 3 \\
\hline 48 & 46 & 35.00 & 34.99 & 35.38 & 34.6 & 34.22 & 35.35 & 35.41 & 35.14 & 34.41 & 50 & 3 \\
\hline 49 & 42,43 & 5.00 & 4.98 & 4.84 & 5.11 & 5.25 & 4.78 & 4.91 & 4.94 & 5.17 & 50 & 3 \\
\hline 50 & $47,48,49$ & 15.00 & 14.89 & 14.67 & 15.13 & 15.36 & 14.36 & 14.96 & 14.85 & 15.19 & 50 & 3 \\
\hline 51 & $47,48,49$ & 25.00 & 25.17 & 25.22 & 25.11 & 25.05 & 25.72 & 24.72 & 25.13 & 25.17 & 0 & 3 \\
\hline 52 & $47,48,49$ & 30.00 & 29.93 & 29.72 & 30.11 & 30.32 & 29.49 & 29.99 & 29.90 & 30.18 & 00 & 3 \\
\hline 53 & $47,48,49$ & 15.00 & 15.02 & 15.03 & 15.02 & 15.01 & 15.11 & 14.96 & 15.02 & 15.02 & 00 & 3 \\
\hline 54 & $47,48,49$ & 15.00 & 15.22 & 15.18 & 15.24 & 15.27 & 15.82 & 14.55 & 15.13 & 15.37 & 0 & 3 \\
\hline 55 & $47,48,49$ & 20.00 & 19.88 & 19.78 & 19.99 & 20.09 & 19.43 & 20.13 & 19.89 & 19.98 & 0 & 3 \\
\hline 56 & $47,48,49$ & 10.00 & 9.92 & 9.87 & 9.98 & 10.04 & 9.64 & 10.09 & 9.93 & 9.97 & 0 & 3 \\
\hline 57 & $50,51,52,53,54,55,56$ & 10.00 & 9.92 & 10.01 & 9.84 & 9.75 & 9.77 & 10.24 & 9.98 & 9.75 & 50 & 3 \\
\hline
\end{tabular}




\begin{tabular}{|c|c|c|c|c|c|c|c|c|c|c|c|}
\hline 58 & $57,59,60$ & 20.00 & 20.16 & 20.44 & 19.89 & 19.6 & 20.92 & 19.95 & 20.2 & 19.82 & 50 \\
\hline 59 & 41 & 5.00 & 4.98 & 5.06 & 4.9 & 4.83 & 4.99 & 5.12 & 5.01 & 4.85 & 0 \\
\hline 60 & 42,43 & 20.00 & 19.91 & 19.82 & 20.01 & 20.10 & 19.57 & 20.07 & 19.92 & 20.01 & 50 \\
\hline 61 & 57,58 & 45.00 & 44.97 & 45.85 & 44.08 & 43.20 & 45.75 & 45.97 & 45.28 & 43.62 & 100 \\
\hline 62 & 61 & 30.00 & 30.18 & 30.79 & 29.59 & 28.99 & 31.36 & 30.21 & 30.32 & 29.38 & 50 \\
\hline 63 & 57 & 30.00 & 30.26 & 30.83 & 29.67 & 29.09 & 31.59 & 30.09 & 30.38 & 29.51 & 50 \\
\hline 64 & 57 & 10.00 & 10.07 & 10.21 & 9.94 & 9.80 & 10.42 & 9.99 & 10.09 & 9.90 & 50 \\
\hline 65 & $61,62,63,64$ & 5.00 & 5.03 & 5.08 & 4.98 & 4.93 & 5.18 & 4.99 & 5.04 & 4.97 & 0 \\
\hline 66 & $61,62,63,64$ & 10.00 & 10.01 & 9.75 & 10.26 & 10.51 & 9.77 & 9.74 & 9.92 & 10.39 & 50 \\
\hline 67 & 66 & 15.00 & 14.80 & 14.62 & 14.99 & 15.17 & 14.03 & 15.21 & 14.81 & 14.98 & 50 \\
\hline 68 & 65,67 & 60.00 & 59.87 & 60.36 & 59.38 & 58.89 & 59.96 & 60.76 & 60.08 & 59.07 & 150 \\
\hline 69 & 68 & 10.00 & 10.1 & 9.94 & 10.25 & 10.41 & 10.23 & 9.65 & 10.01 & 10.38 & 50 \\
\hline 70 & 67 & 30.00 & 29.74 & 29.49 & 29.99 & 30.25 & 28.72 & 30.27 & 29.74 & 29.99 & 100 \\
\hline 71 & 68 & 10.00 & 9.95 & 9.79 & 10.11 & 10.27 & 9.65 & 9.93 & 9.92 & 10.17 & 50 \\
\hline 72 & 68 & 10.00 & 9.98 & 10.17 & 9.78 & 9.59 & 10.09 & 10.24 & 10.05 & 9.68 & 50 \\
\hline 73 & 71,72 & 40.00 & 40.20 & 39.84 & 40.59 & 40.95 & 40.46 & 39.19 & 39.99 & 40.87 & 150 \\
\hline 74 & $68,69,70,73$ & 25.00 & 24.73 & 24.47 & 24.99 & 25.24 & 23.66 & 25.28 & 24.73 & 24.98 & 50 \\
\hline 75 & 74 & 10.00 & 10.00 & 10.00 & 10.00 & 10.00 & 10.00 & 10.00 & 10.00 & 10.00 & 50 \\
\hline 76 & 74 & 10.00 & 10.06 & 10.27 & 9.85 & 9.64 & 10.45 & 10.08 & 10.11 & 9.77 & 100 \\
\hline 77 & 75 & 15.00 & 15.14 & 14.97 & 15.31 & 15.48 & 15.39 & 14.54 & 15.04 & 15.46 & 50 \\
\hline 78 & 79 & 15.00 & 15.13 & 15.01 & 15.25 & 15.37 & 15.39 & 14.62 & 15.04 & 15.38 & 50 \\
\hline 79 & 74 & 15.00 & 14.92 & 14.81 & 15.03 & 15.14 & 14.57 & 15.05 & 14.91 & 15.05 & 50 \\
\hline 80 & $76,77,78$ & 10.00 & 10.08 & 10.30 & 9.85 & 9.63 & 10.53 & 10.08 & 10.13 & 9.78 & 50 \\
\hline 81 & $76,77,78$ & 10.00 & 10.04 & 10.15 & 9.94 & 9.84 & 10.28 & 10.01 & 10.06 & 9.91 & 100 \\
\hline 82 & 80,81 & 10.00 & 10.00 & 10.09 & 9.91 & 9.82 & 10.10 & 10.08 & 10.03 & 9.87 & 0 \\
\hline 83 & 82 & 20.00 & 19.78 & 20.11 & 19.46 & 19.13 & 19.46 & 20.76 & 19.96 & 19.18 & 50 \\
\hline 84 & 83 & 10.00 & 10.01 & 9.96 & 10.06 & 10.12 & 10.01 & 9.92 & 10.00 & 10.10 & 0 \\
\hline 85 & 75,84 & 20.00 & 19.90 & 19.79 & 20.03 & 20.14 & 19.50 & 20.06 & 19.89 & 20.03 & 50 \\
\hline 86 & 82 & 25.00 & 25.04 & 25.04 & 25.06 & 25.07 & 25.18 & 24.89 & 25.02 & 25.08 & 50 \\
\hline 87 & 82 & 20.00 & 20.07 & 20.14 & 20.00 & 19.93 & 20.35 & 19.92 & 20.07 & 20.00 & 50 \\
\hline 88 & 84 & 15.00 & 15.03 & 15.17 & 14.89 & 14.75 & 15.26 & 15.09 & 15.07 & 14.83 & 25 \\
\hline 89 & 88 & 20.00 & 20.30 & 20.43 & 20.15 & 20.01 & 21.30 & 19.57 & 20.25 & 20.23 & 50 \\
\hline 90 & 88 & 30.00 & 29.80 & 29.73 & 29.87 & 29.93 & 29.13 & 30.33 & 29.85 & 29.80 & 50 \\
\hline 91 & $85,86,87,88$ & 20.00 & 19.93 & 20.03 & 19.85 & 19.75 & 19.84 & 20.21 & 19.98 & 19.77 & 50 \\
\hline 92 & $89,90,91$ & 25.00 & 25.01 & 24.94 & 25.10 & 25.17 & 25.00 & 24.88 & 24.99 & 25.14 & 50 \\
\hline 93 & 92 & 10.00 & 9.97 & 9.74 & 10.20 & 10.43 & 9.66 & 9.82 & 9.90 & 10.30 & 50 \\
\hline 94 & $89,90,91$ & 5.00 & 4.99 & 4.94 & 5.05 & 5.11 & 4.91 & 4.96 & 4.98 & 5.08 & 50 \\
\hline 95 & 94 & 20.00 & 19.98 & 19.71 & 20.26 & 20.52 & 19.65 & 19.75 & 19.90 & 20.38 & 50 \\
\hline 96 & $93,95,99$ & 10.00 & 10.08 & 10.22 & 9.94 & 9.81 & 10.46 & 9.98 & 10.10 & 9.92 & 50 \\
\hline 97 & $93,95,99$ & 5.00 & 5.02 & 5.03 & 5.01 & 5.00 & 5.09 & 4.96 & 5.01 & 5.02 & 50 \\
\hline 98 & 92 & 80.00 & 79.62 & 79.08 & 80.21 & 80.74 & 77.99 & 80.12 & 79.52 & 80.28 & 0 \\
\hline 99 & $89,90,91$ & 30.00 & 29.59 & 29.29 & 29.89 & 30.20 & 28.06 & 30.51 & 29.63 & 29.84 & 0 \\
\hline 100 & 98,99 & 10.00 & 10.05 & 10.28 & 9.81 & 9.57 & 10.42 & 10.15 & 10.11 & 9.71 & 50 \\
\hline 101 & 98,99 & 10.00 & 10.04 & 10.08 & 9.99 & 9.95 & 10.18 & 9.98 & 10.04 & 9.99 & 50 \\
\hline 102 & 100,101 & 20.00 & 20.16 & 19.99 & 20.33 & 20.50 & 20.45 & 19.52 & 20.04 & 20.49 & 50 \\
\hline 103 & 100,101 & 30.00 & 29.91 & 29.44 & 30.37 & 30.84 & 29.18 & 29.73 & 29.78 & 30.56 & 50 \\
\hline 104 & 102,103 & 5.00 & 5.02 & 5.03 & 5.01 & 5.01 & 5.09 & 4.97 & 5.02 & 5.02 & 0 \\
\hline 105 & 106 & 30.00 & 30.23 & 30.40 & 30.05 & 29.88 & 31.08 & 29.73 & 30.22 & 30.08 & 50 \\
\hline 106 & 100,101 & 25.00 & 25.08 & 25.28 & 24.89 & 24.69 & 25.53 & 25.03 & 25.11 & 24.83 & 50 \\
\hline 107 & $100,101,104$ & 5.00 & 5.00 & 5.01 & 4.99 & 4.98 & 5.00 & 5.01 & 5.00 & 4.98 & 0 \\
\hline 108 & $100,101,104$ & 5.00 & 5.00 & 4.96 & 5.05 & 5.09 & 4.97 & 4.95 & 4.99 & 5.07 & 0 \\
\hline 109 & 108 & 5.00 & 4.97 & 4.95 & 4.99 & 5.01 & 4.86 & 5.04 & 4.97 & 4.99 & 50 \\
\hline 110 & 108 & 5.00 & 5.05 & 5.08 & 5.02 & 4.99 & 5.24 & 4.94 & 5.05 & 5.03 & 0 \\
\hline 111 & 109,11 & 10.00 & 10.02 & 9.83 & 10.22 & 10.41 & 9.91 & 9.76 & 9.95 & 10.32 & 0 \\
\hline 112 & 109,11 & 10.00 & 9.97 & 9.99 & 9.94 & 9.92 & 9.89 & 10.09 & 9.98 & 9.92 & 0 \\
\hline 113 & 108 & 15.00 & 14.87 & 14.74 & 15.00 & 15.12 & 14.36 & 15.13 & 14.87 & 15.00 & 50 \\
\hline 114 & 113 & 20.00 & 19.84 & 19.75 & 19.94 & 20.03 & 19.28 & 20.21 & 19.86 & 19.91 & 0 \\
\hline 115 & 113 & 20.00 & 19.90 & 19.77 & 20.05 & 20.18 & 19.5 & 20.03 & 19.89 & 20.07 & 0 \\
\hline 116 & $111,112,114,115$ & 45.00 & 44.59 & 44.18 & 44.97 & 45.37 & 42.92 & 45.47 & 44.58 & 44.96 & 100 \\
\hline 117 & 118 & 20.00 & 20.11 & 20.56 & 19.68 & 19.23 & 20.90 & 20.20 & 20.22 & 19.51 & 50 \\
\hline 118 & 116 & 25.00 & 25.38 & 25.23 & 25.53 & 25.68 & 26.38 & 24.09 & 25.20 & 25.80 & 0 \\
\hline 119 & 116 & 25.00 & 24.81 & 24.69 & 24.93 & 25.05 & 24.10 & 25.27 & 24.83 & 24.89 & 0 \\
\hline 120 & 119 & 20.00 & 19.91 & 19.90 & 19.92 & 19.92 & 19.63 & 20.17 & 19.94 & 19.88 & 50 \\
\hline 121 & $105,107,117,120$ & 45.00 & 44.83 & 44.42 & 45.23 & 45.65 & 43.90 & 44.95 & 44.76 & 45.35 & 150 \\
\hline 122 & 121 & 15.00 & 15.08 & 15.19 & 14.97 & 14.86 & 15.43 & 14.95 & 15.09 & 14.96 & 50 \\
\hline 123 & 122 & 10.00 & 9.91 & 9.84 & 9.97 & 10.03 & 9.57 & 10.12 & 9.91 & 9.96 & 50 \\
\hline 124 & 123 & 10.00 & 10.08 & 10.13 & 10.03 & 9.98 & 10.36 & 9.89 & 10.06 & 10.04 & 0 \\
\hline 125 & 124 & 20.00 & 19.97 & 20.29 & 19.64 & 19.33 & 20.19 & 20.39 & 20.09 & 19.47 & 100 \\
\hline 126 & 125 & 30.00 & 29.86 & 29.88 & 29.84 & 29.82 & 29.45 & 30.30 & 29.92 & 29.76 & 50 \\
\hline 127 & 126 & 10.00 & 9.91 & 9.97 & 9.85 & 9.80 & 9.71 & 10.24 & 9.96 & 9.78 & 50 \\
\hline 128 & 117,12 & 25.00 & 24.99 & 25.27 & 24.72 & 24.44 & 25.24 & 25.28 & 25.08 & 24.57 & 50 \\
\hline
\end{tabular}


Postprint : European Journal of Operational Research 251 (2016) 814-829 • http://dx.doi.org/10.1016/j.ejor.2015.12.042

\begin{tabular}{l|l|ccccccccc|c|c}
129 & 126 & 30.00 & 30.02 & 29.68 & 30.34 & 30.68 & 29.71 & 29.66 & 29.91 & 30.52 & 50 & 2 \\
130 & $127,128,129$ & 30.00 & 30.10 & 30.31 & 29.91 & 29.70 & 30.63 & 29.98 & 30.14 & 29.85 & 75 & 2 \\
131 & 117,12 & 40.00 & 40.08 & 40.46 & 39.70 & 39.31 & 40.69 & 40.22 & 40.20 & 39.54 & 50 & 2 \\
132 & 131 & 25.00 & 25.02 & 24.91 & 25.16 & 25.28 & 25.00 & 24.79 & 24.97 & 25.23 & 100 & 1 \\
133 & 130 & 25.00 & 25.15 & 25.46 & 24.81 & 24.5 & 25.89 & 25.05 & 25.22 & 24.73 & 50 & 1 \\
134 & 132 & 20.00 & 20.16 & 19.77 & 20.55 & 20.94 & 20.25 & 19.29 & 19.98 & 20.83 & 50 & 1 \\
135 & 134 & 15.00 & 15.09 & 15.03 & 15.14 & 15.20 & 15.28 & 14.78 & 15.04 & 15.21 & 50 & 1 \\
136 & 135 & 20.00 & 19.99 & 19.81 & 20.17 & 20.35 & 19.80 & 19.83 & 19.94 & 20.26 & 50 & 1 \\
137 & 136 & 30.00 & 29.91 & 29.74 & 30.07 & 30.23 & 29.47 & 30.02 & 29.87 & 30.11 & 50 & 2 \\
138 & 136 & 30.00 & 29.89 & 30.01 & 29.76 & 29.63 & 29.67 & 30.37 & 29.97 & 29.64 & 50 & 2 \\
139 & 137,138 & 15.00 & 14.91 & 14.76 & 15.05 & 15.20 & 14.47 & 15.04 & 14.89 & 15.08 & 100 & 2 \\
140 & 133,139 & 120.00 & 120.93 & 121.60 & 120.2 & 119.53 & 124.33 & 118.93 & 120.91 & 120.33 & 0 & 1 \\
\hline
\end{tabular}

